\title{
TIM3 comes of age as an inhibitory receptor
}

\author{
Yochai Wolf ${ }^{1,2}$, Ana C. Anderson ${ }^{1,2}$ and Vijay K. Kuchroo ${ }^{1,2 *}$
}

Abstract | T cell immunoglobulin and mucin domain-containing protein 3 (TIM3), a member of the TIM family, was originally identified as a receptor expressed on interferon- $\gamma$-producing CD4 ${ }^{+}$ and CD8 ${ }^{+}$T cells. Initial data indicated that TIM3 functioned as a 'co-inhibitory' or 'checkpoint' receptor, but due to the lack of a definable inhibitory signalling motif, it was also suggested that TIM3 might act as a co-stimulatory receptor. Recent studies have shown that TIM3 is part of a module that contains multiple co-inhibitory receptors (checkpoint receptors), which are co-expressed and co-regulated on dysfunctional or 'exhausted' T cells in chronic viral infections and cancer. Furthermore, co-blockade of TIM3 and programmed cell death 1 (PD1) can result in tumour regression in preclinical models and can improve anticancer $T$ cell responses in patients with advanced cancers. Here, we highlight the developments in understanding TIM3 biology, including novel ligand identification and the discovery of loss-of-function mutations associated with human disease. In addition, we summarize emerging data from human clinical trials showing that TIM3 indeed acts as a 'checkpoint' receptor and that inhibition of TIM3 enhances the antitumour effect of PD1 blockade.

Checkpoint receptors Co-inhibitory receptors, mainly on T cells, which are expressed following immune activation to resolve the response and retain homeostasis. Deficits in these receptors might result in autoimmunity, whereas their high expression on tumourinfiltrating T cells can allow tumours to evade elimination.
'Evergrande Center for Immunologic Diseases, Brigham and Women's Hospital, Harvard Medical School, Boston, MA, USA.

${ }^{2}$ Ann Romney Center for Neurologic Diseases, Brigham and Women's Hospital, Harvard Medical School, Boston, MA, USA.

*e-mail:vkuchroo@ evergrande.hms.harvard.edu https://doi.org/10.1038/ s41577-019-0224-6
T cell immunoglobulin and mucin domain-containing protein 3 (TIM3), first discovered in 2002 (REF.'), is a member of the TIM family of immunoregulatory proteins. These are characterized by a common structural organization consisting of an amino-terminal immunoglobulin variable domain ( $\mathrm{V}$ domain) with five noncanonical cysteines, a mucin stalk, a transmembrane domain and a cytoplasmic tail. Members of the TIM family are encoded by three genes in humans (HAVCR1, HAVCR2 and TIMD4, encoding TIM1, TIM3 and TIM4, respectively) and eight genes in mice, located on chromosome band 5q33.2 and chromosome band 11B1.1 in humans and mice, respectively. The genes encoding the TIM family are located within a genomic region that has been associated with allergy and asthma ${ }^{2}$, towards the centromeric end of the IL4, IL5 and IL13 gene locus ${ }^{3}$. Of the TIM family, TIM3 has received the most attention because of its association with the regulation of immune responses in autoimmunity and cancer. Although it was originally identified as a molecule expressed by interferon $-\gamma$ (IFN $\gamma$ )-producing $\mathrm{CD}_{4}^{+}$and $\mathrm{CD}^{+} \mathrm{T}$ cells ${ }^{1}$, many other cell types, including regulatory $\mathrm{T}$ cells $\left(\mathrm{T}_{\text {reg }} \text { cells }\right)^{4}$, myeloid cells $s^{5}$, natural killer (NK) cells ${ }^{6}$ and mast cells ${ }^{7}$, have been shown to express TIM3. Thus, therapeutic targeting of TIM3 likely modulates immune responses by acting on multiple cell types. Blockade of TIM3 is currently being investigated in clinical trials for treatment of cancer alongside the inhibition of checkpoint receptors such as lymphocyte activation gene 3 protein (LAG3) and T cell immunoreceptor with Ig and ITIM domains (TIGIT) ${ }^{8}$. Here, we discuss the advances in our understanding of TIM3 biology.

\section{Molecular mechanism of TIM3 function}

Structure and signalling. A unique feature of TIM3 is its lack of known inhibitory signalling motifs in its cytoplasmic tail (FIG. 1). Unlike more 'classic' checkpoint receptors such as programmed cell death 1 (PD1) and TIGIT, its cytoplasmic tail contains five tyrosines which are conserved between humans and mice. Although the precise intracellular signalling mechanism has not been fully elucidated, it is known that Tyr256 and Tyr263 allow interactions with HLA-B-associated transcript 3 (BAT3) ${ }^{9}$ and the tyrosine kinase $\mathrm{FYN}^{10}$. TIM3 can be found in lipid rafts and is recruited to the immunological synapse on $\mathrm{T}$ cell activation, where it can interact with both BAT3 and the tyrosine kinase LCK $^{11}$. When TIM3 is not bound by a ligand, BAT3 is bound to its cytoplasmic tail and recruits the active, catalytic form of LCK. The current hypothesis is that in this state, TIM3 is permissive to T cell activation. Both the soluble lectin galectin 9 and the adhesion molecule carcinoembyronic antigen-related cell adhesion molecule 1 (CEACAM1), two ligands described for TIM3 (see later), were shown to trigger phosphorylation of Tyr256 and Tyr263 by the tyrosine kinase ITK ${ }^{12,13}$. Upon phosphorylation, BAT3 is released from TIM3, 

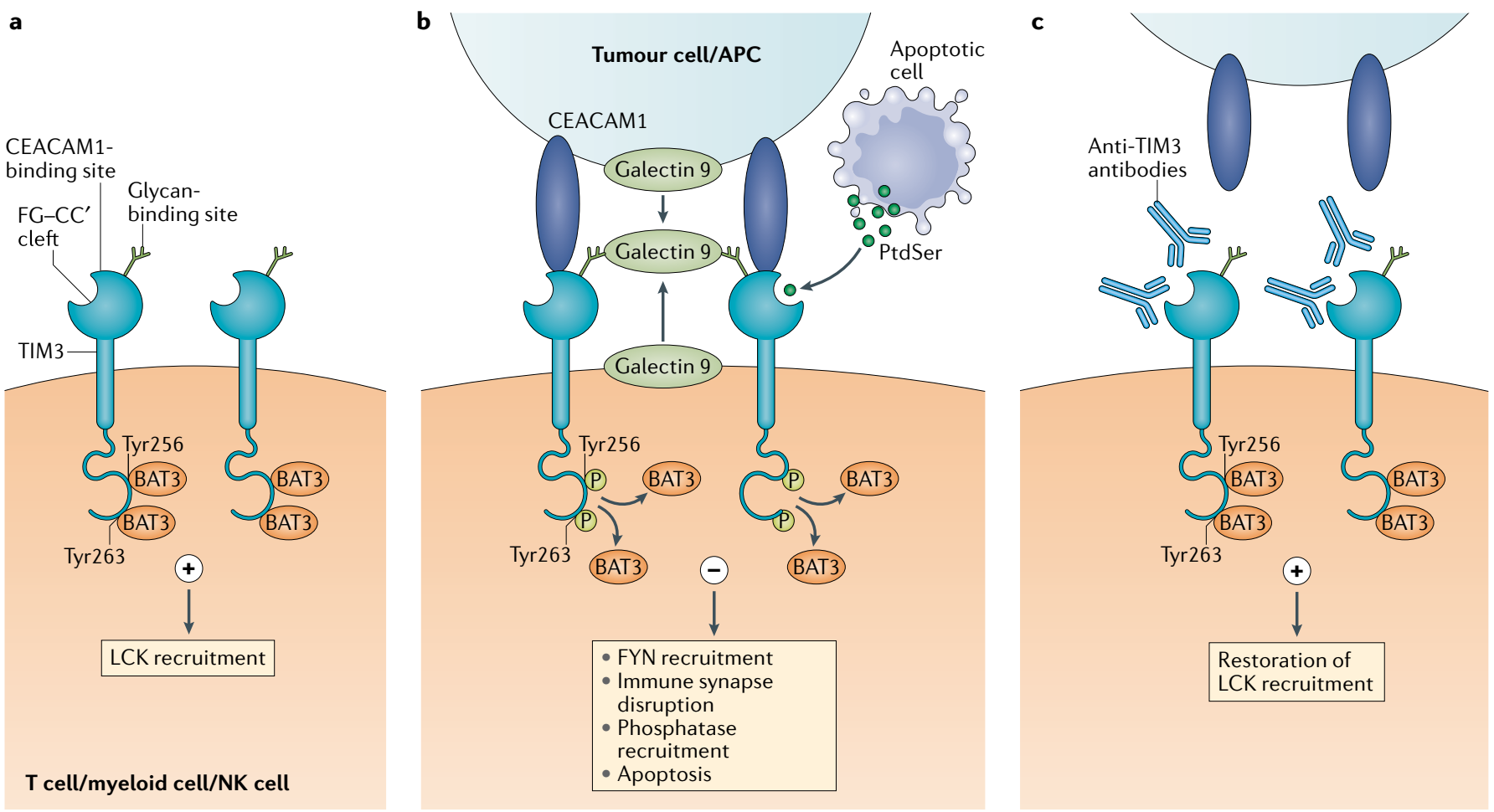

Fig. 1 | Models for TIM3-ligand interactions. a | In its ligand-unbound form, T cell immunoglobulin and mucin domaincontaining protein 3 (TIM3) interacts with HLA-B-associated transcript 3 (BAT3) and maintains T cell activation by LCK recruitment. $\mathbf{b}$ | Galectin 9 can be bound to the surface of tumour cells and can also be secreted by tumour cells, antigenpresenting cells (APCs) and other cells in the parenchyma, or can be secreted in autocrine fashion by TIM3-expressing cells. Its carbohydrate recognition domains bind to the glycan-binding site on TIM3. Galectin 9 has two carbohydrate recognition domains and can promote the oligomerization of TIM3, thus potentially facilitating the formation of other TIM3-ligand complexes such as carcinoembyronic antigen-related cell adhesion molecule 1 (CEACAM1)-TIM3.

In addition, phosphatidylserine (PtdSer) released from apoptotic cells can bind the FG-CC' cleft-binding site on TIM3. On binding to galectin 9 or CEACAM1, Tyr256 and Tyr263 in the intracellular domain of TIM3 are phosphorylated; this releases BAT3 and allows recruitment of the tyrosine kinase FYN. This results in the disruption of immune synapse formation and in phosphatase recruitment. Ultimately, the cell becomes anergic or undergoes apoptosis, which is mediated by intracellular calcium release. $\mathbf{c}$ | Most of the TIM3-targeted antibodies that facilitate antitumour immunity interfere with either CEACAM1 or PtdSer binding to TIM3, thus maintaining the TIM3-BAT3 interaction. NK cell, natural killer cell.

\section{Lipid rafts}

Cholesterol- and sphingolipidenriched microdomains within the plasma membranes in which the assembly of signalling cascade components, such as receptors, kinases and adapter proteins, is localized.

Immunological synapse An interface between a T cell and an antigen-presenting cell, in which the molecular machinery for antigen presentation and T cell activation is present thereby allowing TIM3 to exert its inhibitory function. Bat3-knockout T cells have an elevated expression of co-inhibitory receptors and exhibit reduced pathogenicity in the context of autoimmunity, indicating feedforward negative regulation through BAT3 (REF.'). FYN can potentially contribute to TIM3-mediated inhibitory signalling as it is known to bind the same region of TIM3 as BAT3, indicating potential competition between FYN and BAT3 for TIM3 binding. Moreover, FYN is known to induce $T$ cell anergy by activating phosphoprotein associated with glycosphingolipid-enriched microdomains 1 (PAG), leading to the recruitment of the tyrosine kinase CSK, which then phosphorylates LCK on an inhibitory residue $^{14}$, resulting in the suppression of $\mathrm{T}$ cell receptor (TCR) signalling. TIM3 can also associate with the receptor phosphatases CD45 and CD148 and disrupt the immunological synapse, which has been proposed as an additional mechanism of $\mathrm{T}$ cell inhibition ${ }^{11}$.

On the other hand, ectopic expression of TIM3 in Jurkat cells in vitro in the context of short-term TCR stimulation was shown to promote TCR signalling ${ }^{10}$. In addition, TIM3 expression has been associated with increased AKT-mechanistic target of rapamycin (mTOR) signalling, which was considered a sign of co-stimulation by TIM3 (REF. $\left.{ }^{15}\right)$. These observations led to an ongoing discussion of whether TIM3 is a co-stimulatory or a co-inhibitory receptor, a debate which may be resolved following acquisition of new data regarding the effects of TIM3 loss-of-function mutations in human disease and initial clinical trials with TIM3-blocking antibodies in cancer patients (discussed later).

TIM 3 is also found in a soluble form that lacks the mucin and transmembrane domains. In mice, this form may be generated by alternate splicing ${ }^{16,17}$; however, the existence of this splicing variant in humans is debated ${ }^{18}$. Instead, it has been proposed that soluble TIM3 is a result of metalloproteinase-dependent cleavage that facilitates its shedding from the cell surface ${ }^{19}$. Since elevated plasma levels of TIM3 have been reported in patients with graft-versus-host disease ${ }^{20}$ and in patients with osteosarcoma ${ }^{21}$, soluble TIM3 may serve as a biomarker for these maladies. Elevated plasma levels of TIM3 also appear to correlate with enhanced viral load in patients with HIV infection ${ }^{18}$. However, the exact function of soluble TIM3 is not known at present. 
One can postulate that it may serve as a decoy receptor and act as a 'molecular sink' for TIM3 ligands, thereby interfering with the inhibitory function of TIM3. By contrast, in vitro experiments show that soluble TIM3 can reduce IL-2 production by T cells ${ }^{22}$.

\section{Ligands of TIM3}

TIM3 has been reported to have multiple different ligands (galectin 9, phosphatidylserine (PtdSer), CEACAM1 and high mobility group protein B1 (HMGB1)), which bind to different regions on the TIM3 extracellular immunoglobulin V domain. How TIM3 interacts with each of these ligands and the biological consequences are discussed in the following subsections.

Galectin 9. The first ligand attributed to TIM3 was galectin 9, a C-type lectin broadly expressed and secreted by many haematopoietic cells that binds to carbohydrate moieties on cell surface proteins. On TIM3, it binds to carbohydrate motifs on the immunoglobulin $\mathrm{V}$ domain and can induce intracellular calcium influx and cell death in TIM3 $^{+}$T cells $^{17}$ (FIG. 1). The precise binding site for galectin 9 on TIM3 has not been clearly defined as it requires the prediction of glycosylation sites that are different between mouse and human TIM3; however, predicted residues that can undergo glycosylation in mouse TIM3 include Thr44, Asn100 and Asn74 (REF. ${ }^{23}$ ). Galectin 9 binding results in the oligomerization of TIM3 on the cell surface, inducing the release of BAT3 from the intracellular tail of TIM3. This, in turn, leads to $\mathrm{T}$ cell inhibition and ultimately to cell death'

Multiple lines of evidence highlight the role of the TIM3-galectin 9 interaction in suppressing immune responses. For example, the administration of soluble galectin 9 ameliorated disease in the experimental autoimmune encephalomyelitis (EAE) mouse model of multiple sclerosis, whereas knockdown of galectin 9 expression with siRNA exacerbated disease in this mode ${ }^{17}$. Indeed, in patients with multiple sclerosis, the number of $\mathrm{TIM}^{+}$and galectin 9-positive $\mathrm{CD} 4^{+} \mathrm{T}$ cells in the blood is increased during the benign stages of multiple sclerosis compared with more advanced stages ${ }^{24}$.

In $\mathrm{TIM}^{+} \mathrm{T}_{\text {reg }}$ cells, which have superior suppressor function compared with TIM3 ${ }^{-} \mathrm{T}_{\text {reg }}$ cells, blocking of the TIM3-galectin 9 pathway decreases their suppressive capacity ${ }^{25}$. TIM3 transgenic mice exhibit accelerated tumour growth in an EL4 lymphoma model and decreased severity of disease in an EAE model ${ }^{26}$. Moreover, CT26 colorectal carcinoma tumour cells can secrete galectin 9 and thus inhibit $\mathrm{TIM}^{+} \mathrm{T}$ cells in trans, leading to cell death $^{27}$. In human acute myeloid leukaemia (AML), an autocrine TIM3-galectin 9 loop drives the self-renewal of AML stem cells by activating the nuclear factor- $\mathrm{\kappa B}$ $(\mathrm{NF}-\kappa \mathrm{B})$ and $\beta$-catenin pathways ${ }^{28}$ and by secreting both TIM3 and galectin 9, cancer cells can evade immune surveillance ${ }^{22}$. Moreover, galectin 9 expression may be induced by TIM3 as an inhibitory feedback mechanism. Transgenic mice that overexpress TIM3 in T cells have increased numbers of $\mathrm{CD} 11 \mathrm{~b}^{+} \mathrm{Gr}-1^{+}$myeloid-derived suppressor cells that express galectin 9; moreover, the same increased expansion was seen in mice bearing transgenic galectin 9, and introduction of TIM3 deficiency by crossing galectin 9 transgenic mice with Tim3-knockout mice abrogates this expansion ${ }^{26}$. Collectively, these data show that the TIM3-galectin 9 interaction can act by different mechanisms and on different cell types to suppress immune responses.

The TIM3-galectin 9 interaction is also important in both bacterial and viral infections. During Mycobacterium tuberculosis infection, both lung CD4 ${ }^{+}$ $\mathrm{T}$ cells and lung $\mathrm{CD} 8^{+} \mathrm{T}$ cells express TIM3, which allows them to interact with galectin 9-positive macrophages, leading to a restriction of bacterial proliferation within the macrophages (discussed later) ${ }^{29}$. In the context of HIV infection, in vitro experiments have shown that the binding of galectin 9 to TIM3 on $\mathrm{CD}^{+} \mathrm{T}$ cells lowers the expression of the HIV co-receptors CCR5, CXCR4 and $\alpha 4 \beta 7$ on the T cells, thus enabling them to resist HIV infection $^{30}$. However, the signalling mechanisms in this context are as yet unexplored.

Importantly, there is also evidence that galectin 9 can exert effects that are independent of TIM3. For example, in vitro experiments demonstrated that galectin $9 \mathrm{can}$ enhance cytokine production in both $\mathrm{T}$ helper 1 cells $\left(\mathrm{T}_{\mathrm{H}} 1\right.$ cells) and $\mathrm{T}_{\mathrm{H}} 2$ cells ${ }^{31}$ and suppress $\mathrm{T}_{\mathrm{H}} 17$ cell differentiation. This was independent of TIM3 expression but required $O$-linked glycosylation of an unknown glycoprotein and IL-2 (REF. ${ }^{32}$ ). By virtue of binding $\mathrm{N}$-linked sugars of a defined configuration, galectin 9 can also bind to the cell-surface glycoprotein CD44 on $\mathrm{T}_{\text {reg }}$ cells and thereby regulate their function ${ }^{33}$. On the basis of binding assays and functional assays performed with HIV-1-infected human T cells, some investigators have also argued that TIM3 does not bind galectin 9 $\left(\mathrm{REF}^{34}\right)$, which is in stark contrast to most of the TIM3 literature. Since galectin 9 does not bind in the unique pocket framed by the FG and CC' loops of TIM3, it was predicted that TIM3 must have additional ligands that bind to this pocket ${ }^{23}$.

Phosphatidylserine. The elucidation of the crystal structures of TIM1, TIM3 and TIM4 led to the discovery of PtdSer, a phospholipid that acts as a surface marker for apoptotic cells (reviewed in REF. ${ }^{35}$ ), as a ligand for all TIM family members ${ }^{36,37}$. PtdSer binds to the pocket framed by the FG and CC' loops in the TIM3 immunoglobulin $\mathrm{V}$ domain ${ }^{37}$, with coordinated calcium binding $^{23}$ involving the amino acids Asn119 and Asp120 (REF. ${ }^{38}$ ). However, TIM3 binds PtdSer with at least five times lower affinity compared with other TIM family members $^{39}$. Notably, the binding site for PtdSer is on the opposite side of the immunoglobulin $\mathrm{V}$ domain relative to the predicted sites for galectin 9 binding $^{35}$ and appears to be affected by allelic polymorphisms as $\mathrm{BALB} / \mathrm{c}$ allelic variants have a stronger capacity to bind PtdSer than congenic C.D2 Es-Hba variants ${ }^{37}$. Although binding of PtdSer to TIM3 does not lead to engulfment and phagocytosis of apoptotic material by $\mathrm{T}$ cells, it is postulated that it is important for antigen cross-presentation by $\mathrm{TIM}^{+}$dendritic cells (DCs) ${ }^{39}$. Importantly, all mouse and human anti-TIM3 antibodies that have demonstrated functional efficacy in vivo and in vitro interfere with TIM3 binding to PtdSer and also to CEACAM1 (discussed later) ${ }^{40}$. 


\section{Alarmin}

A broad definition of molecules released from dying cells that serve as a danger signal for immune cells.
HMGB1. The alarmin HMGB1 was reported as a ligand for $\mathrm{TIM}^{+} \mathrm{DCs}$, but its binding site has not been determined $^{41}$. HMGB1 binds to DNA released from dying cells and facilitates nucleic acid sensing by Toll-like receptors (TLRs). HMGB1 can also be secreted by tumour cells ${ }^{42}$. Thus, TIM3 ${ }^{+}$myeloid cells in the tumour microenvironment may act as a molecular sink for HGMB1, thereby interfering with its function in innate immune activation (FIG. 2). Indeed, in models of MC38 colon carcinoma TIM3 blockade was shown to increase both the efficacy of DNA-based tumour vaccines and responsiveness to cisplatin $^{41}$, which is known to trigger release of HMGB1 $\left(R E F{ }^{43}\right)$. This effect was unchanged by the administration of galectin 9-binding antibodies or annexin $\mathrm{V}$, which binds PtdSer, suggesting that the HMGB1-TIM3 interaction is independent of other TIM3 ligands. It will be interesting to test whether TIM3 blockade also increases the efficacy of other chemotherapeutic drugs that trigger HMGB1 release. Whether these effects depend on the interaction of TIM3 with HMGB1 or with PtdSer displayed on the surface of dying cells remains to be addressed. Although intriguing, there has been little follow up of the TIM3-HMGB1 interaction and its relevance to TIM3-mediated regulation beyond the initial report ${ }^{41}$.

CEACAM1. The most recently discovered ligand for TIM3 is the glycoprotein CEACAM1 (REFS ${ }^{13,44}$ ), which is known to have a role in the regulation of antiviral responses ${ }^{45}$. In vitro, CEACAM1 is co-expressed with TIM3 on ovalbumin peptide-stimulated and Staphylococcus aureus endotoxin B-stimulated T cells following repeated exposure to these antigens, and is therefore thought to contribute to establishing $\mathrm{T}$ cell tolerance ${ }^{13}$.

CEACAM1 is thought to bind to the $\mathrm{CC}^{\prime}$ and FG loops of TIM3 $\left(\right.$ REF $\left.^{13}\right)$. CEACAM1 has also been found to be able to bind TIM3 intracellularly, which appears to be important for the maturation of TIM3, as mutant forms of either TIM3 or CEACAM1 co-expressed in HEK293 cells resulted in intracellular TIM3 accumulation and TIM3 hypoglycosylation ${ }^{13}$. Accordingly, in a mouse model of colitis, CEACAM1 ${ }^{-1-} \mathrm{T}$ cells expressed reduced surface levels of TIM3 concomitant with greater production of the effector cytokines IFN $\gamma$, tumour necrosis factor (TNF), and IL-17A ${ }^{13}$. CEACAM1

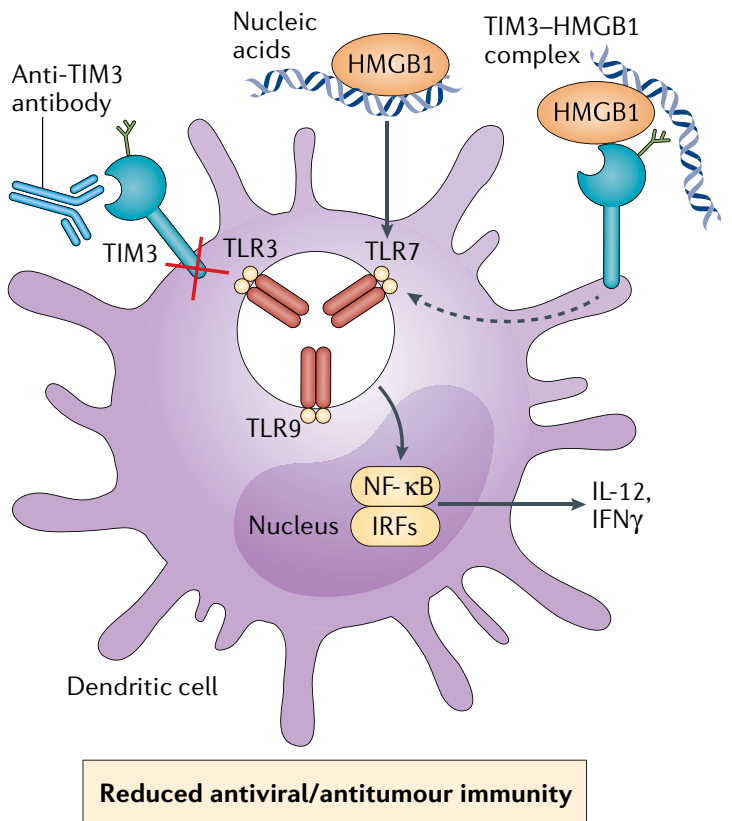

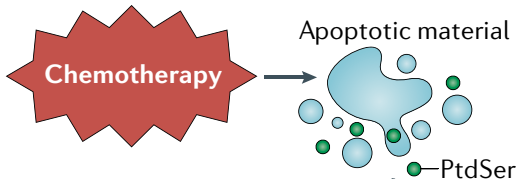

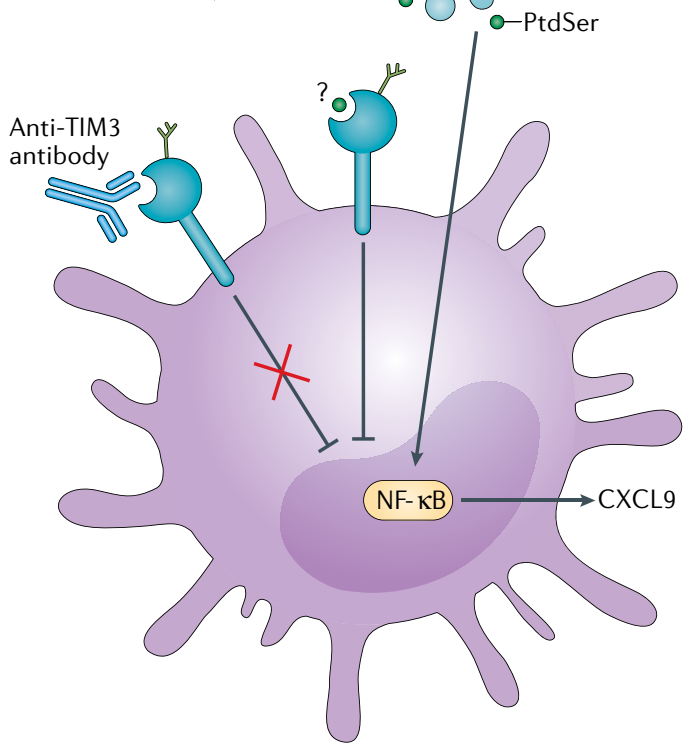

Indirect reduced $\mathrm{CD}^{+} \mathrm{T}$ cell cytotoxicity

Fig. 2 | TIM3 function in dendritic cells. a | Endosomal Toll-like receptor 3 (TLR3), TLR7 and TLR9 sense viral doublestranded RNA, single-stranded RNA and bacterial or viral DNA, respectively, and activate several downstream transcription factors, such as nuclear factor- $\mathrm{KB}$ (NF-kB) and several interferon regulatory factors (IRFs), giving rise to the secretion of IL-12 and interferon- $\gamma$ (IFN $\gamma$ ). In dendritic cells, the activation of TLR3, TLR7 and TLR9 can be suppressed by $T$ cell immunoglobulin and mucin domain-containing protein 3 (TIM3)-mediated sequestration of high mobility group protein B1 (HMGB1), a protein that binds nucleic acids and facilitates their uptake, leading to TLR signalling. Thus, TIM3 serves as a molecular sink and prevents the entry of the nucleic acids into the cell, thereby dampening both antiviral and antitumour immune responses. Antibody-mediated blockade of TIM3 can relieve this suppression. b | TIM3 is highly expressed in type 1 conventional dendritic cells in tumours, where its blockade promotes the expression of CXC-chemokine ligand 9 (CXCL9) by type 1 conventional dendritic cells and the recruitment of CD8 ${ }^{+} T$ cells that can respond to tumour antigens released by chemotherapy-induced apoptosis. More findings on TIM3 function in dendritic cells and other non-T cell leukocytes are described in TABLE 1. PtdSer, phosphatidylserine. 
binding can trigger the release of BAT3 from TIM3, thus allowing TIM3-mediated inhibition of TCR signalling ${ }^{13}$. In addition to its expression by $\mathrm{T}$ cells, CEACAM1 is expressed by DCs ${ }^{46}$, monocytes ${ }^{47}$ and macrophages ${ }^{48}$. Thus, the TIM3-CEACAM1 axis can potentially inhibit immune responses either in cis or in trans in both T cells and myeloid cells. The cis interaction promotes the stability of mature TIM3 on the cell surface, and both cis and trans interactions mediate the inhibitory function of TIM3.

The TIM3-CEACAM1 axis has the potential to be an important target for immunotherapy for cancer. As noted earlier, all antibodies directed against mouse TIM3 that have demonstrated functional efficacy in vivo in mouse cancer models interfere with both PtdSer binding and CEACAM1 binding ${ }^{40}$. CEACAM1 is also highly expressed by some tumour cells, such as melanoma cells, where it is thought to dampen $\mathrm{T}$ cell responses ${ }^{49}$. However, CAECAM1 may also have tumour-suppressive function under some circumstances, since it was found that CEACAM1 ${ }^{-/-}$mice develop a higher tumour burden in a chemically induced model of colorectal cancer ${ }^{50}$. Thus, TIM3-CEACAM1 interactions in the tumour microenvironment are highly complex and require careful molecular dissection to delineate regulatory mechanisms.

Multiple ligand interactions. Notably, all anti-TIM3 antibodies with antitumour activity have the capacity to interfere with PtdSer and CEACAM1 binding but not galectin 9 binding to TIM3 $\left(\mathrm{REF}^{40}\right)$. This leaves open the question of whether and how galectin 9 may play a role in TIM3 signalling in tumours. It has been shown that galectin 9, which has two carbohydrate recognition domains, can form a molecular bridge between TIM3 complexed with other ligands, such as CEACAM1 and PtdSer, and the phosphatases CD45 and CD148 at the immunological synapse ${ }^{11}$.

Given the variety of TIM3 ligands, it is difficult to speculate as to how $\mathrm{TIM}^{+}$cells can discriminate between these ligands. Since the affinity of TIM3 for all of its ligands is unknown at present, it is possible that the distribution and individual expression level of each ligand in a given tissue microenvironment determines the signalling through TIM3. Indeed, some tissues, such as those of the gastrointestinal tract and specifically the colon, contain cells that express high levels of CEACAM1 (REF. ${ }^{51}$ ), whereas others, such as those of the liver, are enriched in cells expressing galectin 9 ( REFS $^{52,53}$ ). Notably, galectin 9 binding does not preclude binding to other ligands in different binding sites. Moreover, we predict that galectin 9 binding might facilitate TIM3 binding to other ligands by inducing an allosteric change that makes the cleft framed by the FG and $\mathrm{CC}^{\prime}$ loops more accessible to other ligands (FIG. 1).

\section{The functions of TIM3 in immune regulation} Role of TIM3 in transplant tolerance and autoimmunity. TIM3 was initially identified as a marker for IFN $\gamma$ secreting effector $\mathrm{CD} 4^{+} \mathrm{T}_{\mathrm{H}} 1$ cells and $\mathrm{CD} 8^{+}$type 1 cytotoxic $\mathrm{T}$ cells ${ }^{1}$, and was later found to be expressed by human $\mathrm{T}_{\mathrm{H}} 1$ cells $\mathrm{s}^{54}$. The $\mathrm{T}_{\mathrm{H}} 1$ cell transcription factor
T-bet was found to regulate TIM3 expression ${ }^{55}$ in cooperation with other transcriptional regulators, including NFIL3 (REF. ${ }^{56}$ ), PRDM1 and MAF ${ }^{57}$. Treatment of mice with TIM3-blocking antibodies or a soluble TIM3immunoglobulin fusion protein, which acts as a molecular sink for TIM3 ligands, exacerbates disease in the EAE model and results in enhanced $\mathrm{T}_{\mathrm{H}} 1$ cell immunity and macrophage hyperactivation. This gave the first indication that TIM3 might function as an inhibitory molecule that serves to limit IFN $\gamma$-driven inflammation. In keeping with the expression of TIM 3 on IFN $\gamma$-secreting $\mathrm{T}_{\mathrm{H}} 1$ cells, TIM3 deficiency leads to $\mathrm{T}_{\mathrm{H}} 1$ cell dysregulation but not $\mathrm{T}_{\mathrm{H}} 17$ cell dysregulation in $\mathrm{EAE}^{58}$. Later it was confirmed that TIM3 is also expressed in human $\mathrm{T}_{\mathrm{H}} 17$ cells, albeit to a lesser extent relative to $\mathrm{T}_{\mathrm{H}} 1$ cells $\mathrm{s}^{59}$. Notably, the surface expression of TIM3 on T cells is low in patients with ulcerative colitis ${ }^{60}$, multiple sclerosis ${ }^{61,62}$, rheumatoid arthritis ${ }^{63}$ and psoriasis ${ }^{64}$ compared with that on T cells from healthy controls; this is consistent with the limited regulation of the inflammatory properties of these $\mathrm{T}$ cells in patients with these autoimmune diseases. Indeed, TIM3 blockade is known to worsen disease in multiple preclinical models of autoimmune inflammation, including inflammatory bowel disease ${ }^{65}$ and diabetes $^{66}$. Moreover, both TIM3-deficient mice and mice treated with a TIM3-immunoglobulin fusion protein exhibit defects in the induction of antigen-specific tolerance in two different models: administration of highdose soluble antigen and transfusion of allogeneic donor splenocytes together with CD154 (CD40L) blockade ${ }^{16,66}$. In line with these data, administration of galectin 9 suppresses allogenic skin graft rejection ${ }^{67}$.

It is now recognized that TIM3 is also expressed by FOXP3 ${ }^{+} \mathrm{T}_{\text {reg }}$ cells, specifically those at tissue sites. In vitro experiments have shown that $\mathrm{TIM}^{+} \mathrm{T}_{\text {reg }}$ cells are enriched in effector molecules such as IL-10, perforin, and granzyme $\mathrm{A}^{53,68,69}$ and have superior suppressive function when compared with TIM3 ${ }^{-} \mathrm{T}_{\text {reg }}$ cells ${ }^{68,69}$. Indeed, $\mathrm{TIM}^{+} \mathrm{T}_{\text {reg }}$ cells appear to be instrumental in the suppression of allograft rejection as their numbers in draining lymph nodes and spleens are significantly increased following allogeneic but not syngeneic grafts. They also show a more suppressive phenotype, as well as higher proliferation rate, compared with allograftinduced TIM3- $\mathrm{T}_{\text {reg }}$ cells ${ }^{69}$. An open question is whether $\mathrm{TIM}^{+} \mathrm{T}_{\text {reg }}$ cells are highly active effector $\mathrm{T}_{\text {reg }}$ cells or are a unique subset of $\mathrm{T}_{\text {reg }}$ cells with specialized effector functions directed at distinct cell types or tissue sites.

Role of TIM3 in M. tuberculosis infection. Infection with $M$. tuberculosis remains a serious threat to human health. During chronic M. tuberculosis infection in mice, TIM3 expression on CD8 ${ }^{+} \mathrm{T}$ cells is elevated ${ }^{70,71}$. Similarly, elevated TIM3 expression is found on total $\mathrm{CD}^{+} \mathrm{T}$ cells and antigen-specific $\mathrm{CD} 8^{+} \mathrm{T}$ cells in patients with tuberculosis ${ }^{72,73}$. In vivo administration of TIM3-immunoglobulin fusion protein reduced the M. tuberculosis burden in M. tuberculosis-infected mice $^{29}$. Further in vitro analyses demonstrated that $\mathrm{TIM}^{+} \mathrm{T}$ cells interact with galectin 9 on macrophages, which triggers IL- $1 \beta$ production by macrophages and thereby limits intracellular M. tuberculosis replication 
in macrophages ${ }^{29,74}$. This is likely mediated by a yet unknown, indirect mechanism, as galectin 9 itself does not contain a signalling motif. A similar role for the TIM3-galectin 9 pathway in triggering antibacterial activity in $M$. tuberculosis-infected human macrophages has been identified ${ }^{75}$. Thus, during $M$. tuberculosis infection, TIM3 seems to have a dual role, mediating the activation of macrophages by galectin 9 to inhibit intracellular bacterial replication while restraining TIM3 $^{+}$ effector T cell responses.

Role of TIM3 in cancer and chronic viral infections. A key obstacle to successful antitumour immunity is $\mathrm{T}$ cell dysfunction or $\mathrm{T}$ cell exhaustion, a cellular state characterized by compromised proinflammatory cytokine production and cytotoxicity that develops in response to continuous exposure to antigen, most notably in chronic viral infections and cancer ${ }^{76}$. TIM3 serves as a marker of a highly dysfunctional subset of CD8 ${ }^{+}$ $\mathrm{T}$ cells in patients infected with HIV, and in vitro experiments showed that TIM3 blockade with antibodies can restore the proliferative response of $\mathrm{TIM}^{+} \mathrm{CD}^{+} \mathrm{T}$ cells to $\mathrm{HIV}-1$ peptides ${ }^{77}$. Subsequently, TIM3 expression was found to be a marker for the most dysfunctional $\mathrm{T}$ cell population within the $\mathrm{CD} 8^{+} \mathrm{PD} 1^{+} \mathrm{T}$ cell pool in humans with chronic viral infections (hepatitis $\mathrm{C}$ virus infection, hepatitis $\mathrm{B}$ virus (HBV) infection) and also in mouse models of infection (lymphocytic choriomeningitis virus infection, HBV infection, Friend virus infection $)^{78-81}$. Moreover, TIM3 levels are positively correlated with viral load and disease progression in many viral infections, which is consistent with its proposed inhibitory role ${ }^{77,78,82}$.

TIM3 also marks the most dysfunctional subset among tumour-infiltrating $\mathrm{CD} 8{ }^{+} \mathrm{PD} 1^{+} \mathrm{T}$ cells in can$\operatorname{cer}^{79,83,84}$. Accordingly, antibody blockade of both TIM3 and PD1 was shown to have a synergistic effect with regard to inhibiting tumour growth ${ }^{84}$ and improving tumour antigen-specific $\mathrm{CD}^{+} \mathrm{T}$ cell responses of patient-derived T cells ${ }^{83,85}$. In contrast, TIM3 overexpression on T cells exacerbates tumour progression in the EL4 mouse model of lymphoma ${ }^{26}$. In addition to $\mathrm{TIM}^{+} \mathrm{CD}^{+}{ }^{+} \mathrm{T}$ cells, $\mathrm{TIM}^{+} \mathrm{T}_{\text {reg }}$ cells might also be targeted by anti-TIM3 antibody therapy as $\mathrm{TIM}^{+} \mathrm{T}_{\text {reg }}$ cells are the predominant $\mathrm{T}_{\text {reg }}$ cell population in tumours ${ }^{4,53,68}$ and their presence correlates with tumour severity and progression $^{4}$. For example, in patients with lung cancer, $60 \%$ of $\mathrm{T}_{\text {reg }}$ cells were found to express TIM3 (REF.), and in patients with hepatocellular carcinoma, $70 \%$ of $\mathrm{T}_{\text {reg }}$ cells were found to express TIM3 (REF.53). In a mouse model of colorectal cancer, $\mathrm{TIM}^{+} \mathrm{T}_{\text {reg }}$ cells accounted for more than $50 \%$ of total $\mathrm{T}_{\text {reg }}$ cells, a proportion that far exceeds their relative frequency in tumour-draining lymph nodes, spleen or blood. Moreover, in tumour models such as the MC38 colon carcinoma mouse model, the vast majority of $\mathrm{CD}^{+}{ }^{+} \mathrm{TIM}^{+}{ }^{+} \mathrm{T}$ cells also expressed FOXP3 ( 90\%), indicating that they are $\mathrm{T}_{\text {reg }}$ cells ${ }^{68}$. Indeed, TIM3 blockade in combination with PDL1 blockade results in a downregulation of $\mathrm{T}_{\text {reg }}$ cell effector molecules in $\mathrm{TIM}^{+} \mathrm{T}_{\text {reg }}$ cells in vivo ${ }^{68}$, and TIM3 blockade lowered both TIM $^{+}{ }^{+} \mathrm{T}_{\text {reg }}$ cell numbers and tumour growth in a mouse model of head and neck cancer ${ }^{86}$.
TIM3 can also serve as a prognostic marker for solid tumours such as colon, gastric and cervical cancer, nonsmall-cell lung cancer and clear cell renal carcinoma, with high levels of TIM3 expression associated with lower survival and vice versa ${ }^{87}$. Furthermore, elevated TIM3 levels on cells in both the circulation and the tumour tissue of patients with colorectal cancer correlated with an unfavourable disease course ${ }^{88}$. Similar findings were obtained in patients with $\mathrm{HBV}$-associated hepatocellular carcinoma, where survival is negatively correlated with the presence of $\mathrm{TIM}^{+} \mathrm{T}$ cells ${ }^{89}$. In patients with hepatocellular carcinoma negative for HBV, TIM3 expression on both CD4 $4^{+} \mathrm{T}$ cells and CD8 ${ }^{+}$ $\mathrm{T}$ cells is minimal ${ }^{89}$.

TIM3 expression is also observed on malignant haematopoietic cells, specifically AML cells (reviewed in REF ${ }^{90}$ ). TIM3 is highly expressed by leukaemic stem cells but not by normal haematopoietic stem cells in these tumours ${ }^{91-93}$. Most of the disease-initiating cells appear to express TIM3, since xenograft models show that $\mathrm{TIM}^{+}$cells from patients with AML, but not TIM3 cells, can establish AML when administered to immunodeficient mice ${ }^{94}$. The exact role of TIM3 on leukaemic stem cells is not known; however, anti-TIM3 antibody blockade can ameliorate disease in a xenograft model of AML, indicating that TIM3 can act as both a biomarker and a clinical target ${ }^{94}$.

One feature of TIM3 that makes it an attractive target for checkpoint-targeted cancer immunotherapy is its co-expression with PD1 (REFS ${ }^{83,84}$ ). In preclinical models, co-expression of TIM3 and PD1 is observed in both $\mathrm{CD}^{+} \mathrm{T}$ cells and $\mathrm{CD} 8^{+} \mathrm{T}$ cells and $\mathrm{TIM} 3^{+} \mathrm{PD} 1^{+} \mathrm{T}$ cells appear to be most dysfunctional, expressing significantly lower amounts of the effector cytokines IFN $\gamma$, TNF and IL-2 compared with their TIM3 ${ }^{-}$counterparts $^{84}$. Moreover, TIM3 can be upregulated as a result of PD1directed therapy; this has been shown to be mediated by the phosphoinositide 3-kinase (PI3K)-AKT pathway ${ }^{95}$. Co-blockade of the TIM3 and PD1 pathways results in greater restoration of $\mathrm{T}$ cell responses than PD1 pathway blockade alone in mouse models of melanoma, colorectal cancer and $\mathrm{AML}^{84,96-98}$, with similar outcomes in mouse models of hepatitis $\mathrm{C}$ virus infection, $\mathrm{HBV}$ infection, and lymphocytic choriomeningitis virus infection $^{78,79,99,100}$.

The immunosuppressive cytokine IL-27 was recently shown to be one of the key drivers of TIM3 expression on both $\mathrm{CD}^{+} \mathrm{T}$ cells and $\mathrm{CD}^{+} \mathrm{T}$ cells ${ }^{56}$. IL-27 induces transcriptional regulators that activate a module of coinhibitory receptors that includes not only PD1 and TIM3 but also TIGIT and LAG3 (REF. ${ }^{57}$ ). Thus, there is a common link between TIM3 and other checkpoint receptors that regulate effector $\mathrm{T}$ cell function. As a single agent, TIM3-blocking antibodies do not have a substantial clinical benefit; however, they elicit stronger immune responses when combined with other checkpoint molecules. The advantage of targeting TIM3 with checkpoint inhibitors is that this may circumvent some of the toxic effects that have been observed with CTLA4- or PD1-directed checkpoint blockade, since its expression on $\mathrm{T}$ cells is limited to terminally differentiated IFN $\gamma$-producing T cells, unlike CTLA4 and PD1, 
Table 1 | The role of TIM3 in non-T cell leukocytes

\begin{tabular}{|c|c|}
\hline Cell type & Functions of TIM3 \\
\hline $\mathrm{CD} 8^{+} \mathrm{DCs}$ & $\begin{array}{l}\text { Facilitates the uptake of apoptotic material and enhances } \\
\text { antigen cross-presentation }{ }^{39}\end{array}$ \\
\hline \multirow{2}{*}{$\begin{array}{l}\text { Tumour-associated } \\
\text { DCs }\end{array}$} & Inhibits the activation of nucleic acid sensing TLRs ${ }^{41}$ \\
\hline & $\begin{array}{l}\text { Reduces CXCL9 secretion by tumour cDC1s and dampens } \\
\text { CD8 }{ }^{+} \text {T cell cytotoxicity indirectly }{ }^{119}\end{array}$ \\
\hline \multirow[t]{4}{*}{ Macrophages } & $\begin{array}{l}\text { Regulates the response to TLR2 and TLR4 stimulation; } \\
\text { is upregulated on macrophages in sepsis and downregulated } \\
\text { in severe sepsis }{ }^{113}\end{array}$ \\
\hline & Inhibits the release of proinflammatory cytokines in DSS colitis ${ }^{114}$ \\
\hline & Interacts with STAT1, which leads to miR-155 expression ${ }^{116}$ \\
\hline & $\begin{array}{l}\text { Interacts with NRF2, leading to reduced expression of CD36 } \\
\text { and the haem oxygenase } 1-\mathrm{IL}-1 \beta \text { pathway; in experimental } \\
\text { Listeria monocytogenes infection, this results in reduced } \\
\text { ingestion of bacteria }\end{array}$ \\
\hline Mast cells & $\begin{array}{l}\text { Mediates mast cell activation following lgE sensitization and } \\
\text { antigen-dependent activation }\end{array}$ \\
\hline \multirow[t]{2}{*}{ NK cells } & May promote dysfunction in cancer $r^{6,127}$ \\
\hline & Induces maternal-fetal tolerance ${ }^{130-132}$ \\
\hline
\end{tabular}

which are globally expressed on all $\mathrm{T}$ cells on activation. Moreover, TIM3 expression is restricted to intratumoural $\mathrm{T}_{\text {reg }}$ cells, whereas CTLA4 is broadly expressed by all $\mathrm{T}_{\text {reg }}$ cells. Finally, both CTLA4-deficient mice ${ }^{10,102}$ and PD1-deficient mice ${ }^{103,104}$ develop spontaneous autoimmunity, whereas TIM3-deficient mice do not. Thus, blockade of TIM3 in combination with CTLA4 or PD1 is likely to be both safer and more specifically targeted to intratumoural $\mathrm{T}$ cells than the combination of CTLA4 and PD1 blockade. Recently, co-blockade of TIM3 and the ectonucleotidase CD39, with a TIM3-blocking antibody and the small molecule POM-1, was shown to significantly reduce tumour growth in the B16F10 melanoma mouse model. Both TIM3 and CD39 were found be highly expressed by $\mathrm{CD}^{+} \mathrm{T}$ cells from cancer patients who were unresponsive to anti-PD1 therapy ${ }^{105}$, suggesting that TIM3-blocking antibodies can be used as cancer therapeutics in combination with drugs other than anti-PD1 antibodies.

In line with the observation that TIM3 is a marker for the most dysfunctional $\mathrm{T}$ cell subset in both cancer and chronic viral infection, it has recently been noted that TIM3 expression is a marker for T cells that have lost the expression of Tcf7, which encodes TCF1, a transcription factor that maintains stemness and restrains effector differentiation ${ }^{106}$. Thus, there is a negative correlation between TIM 3 and TCF1 expression, where TCF1 expression can serve as a marker for stem-like cells and TIM3 expression can serve as a marker for terminally differentiated effector and exhausted cells that cannot be reinvigorated by checkpoint blockade ${ }^{106-111}$. Indeed, the presence of $\mathrm{PD}^{-} \mathrm{T}$ cells and $\mathrm{PD} 1^{+} \mathrm{T}$ cells that express TCF1 is essential for the response to checkpoint blockade $^{107,109}$. Whether there is a regulatory relationship between TIM3 and TCF1 and whether this occurs at the transcriptional or post-translational level is currently not known.

\section{Role of TIM3 in non-T cells}

Although it was initially thought that TIM3 is expressed only by T cells, it is now appreciated that TIM3 is expressed by multiple cell types, including DCs, macrophages, myeloid-derived suppressor cells and NK cells (TABLE 1). Although the role of TIM3 in some of these cell types is still being investigated, accumulating data indicate that TIM3 also functions as an inhibitory receptor in these cells. The current knowledge of TIM 3 biology in these non-T cell populations is limited.

There appears to be a close connection between TIM3 expression and the modulation of TLR activation in myeloid cells. In vitro experiments showed that TIM3 antibody blockade or TIM3 knockdown with siRNA in monocytes and macrophages that were stimulated through multiple different TLRs increases the production of many cytokines and the expression of TLR2 and TLR4, indicating that TIM3 is a negative regulator of the TLR respons $\mathrm{e}^{112,113}$. Accordingly, TIM3 blockade can worsen experimental sepsis in wild-type but not $\mathrm{Tlr}^{-/-}$mice $^{113}$. In a model of colitis induced by dextran sodium sulfate treatment, colonic macrophages expressed lower levels of TIM3 and showed increased macrophage polarization towards a proinflammatory response, with excessive production of TNF, IL-1 $\beta$, NOS2, IL-6 and IL-12. In contrast, overexpression of TIM3 in macrophages both reduced the expression of genes encoding proinflammatory mediators and attenuated colitis in this model ${ }^{114}$. TIM3 overexpression on macrophages appears to attenuate the massive inflammatory response to dextran sodium sulfate and thereby prevent undesired tissue disease. In line with this, TIM3 expression is found to be upregulated on peripheral blood mononuclear cells in patients with acute sepsis but is found to be suppressed in patients with severe sepsis ${ }^{113}$. TIM3 is also highly expressed on human peripheral blood mononuclear cells during pregnancy, where TIM3 appears to blunt the response to TLR4 stimulation $^{115}$. An analysis of how TIM3 inhibits signalling through TLR4 suggested a mechanism of NF- $\mathrm{KB}$ inhibition that is dependent on the activation of the PI3K-AKT pathway and the NF- $\kappa B$ inhibitor protein A20 (REF. ${ }^{13}$ ). Lastly, as discussed earlier, TIM3 expression on DCs may inhibit nucleic acid sensing through TLRs ${ }^{41}$.

TIM3 has also been reported to have TLR-independent effects in macrophages. TIM3 can act as an adaptor protein for the transcription factor STAT1 (signal transducer and activator of transcription 1), which binds to Tyr256 and Tyr263 of the intracellular tail of TIM3. This leads to the suppression of transcription of the inflammatory microRNA miR-155 and enhances the function of SOCS1, a negative regulator of Janus kinase (JAK) signalling. This, in turn, promotes M2 macrophage polarization ${ }^{116}$. TIM3 has also been shown to interact with the transcription factor NRF2 (nuclear factor erythroid 2-related factor 2), which leads to the downmodulation of the scavenger protein CD36 and the haem oxygenase $1-$ IL-1 $\beta$ pathway. In a model of Listeria monocytogenes infection, this results in lower levels of bacterial ingestion by macrophages ${ }^{117}$.

Given that TIM3 mediates the capture of apoptotic material by binding to PtdSer, its expression on mouse macrophages and $\mathrm{CD} 8^{+}$DCs can facilitate 
cross-presentation $^{39}$. Of note, other members of the TIM family, such as TIM1 and TIM4 (the latter being highly expressed on several tissue-resident macrophages and DCs), have a much higher affinity for PtdSer and thus are more potent in allowing the uptake of apoptotic material $^{118}$. The lower binding affinity of TIM3 for PtdSer is because TIM3 has only two of the four conserved residues in the FG and $\mathrm{CC}^{\prime}$ loops that mediate the interaction with PtdSer ${ }^{35,37}$. How the roles of TIM3 in antigen cross-presentation ${ }^{39}$ and suppression of myeloid cell responses to inflammatory stimuli ${ }^{117}$ relate to each other is incompletely understood. Which one of these functions dominates could be a critical determinant of $\mathrm{T}$ cell activation and disease outcome.

A recent study showed that TIM3 blockade improved the antitumour response to chemotherapy in a mouse model of breast cancer ${ }^{119}$. Anti-TIM3 antibodies promoted the production of the chemokine CXCL9 by $\mathrm{BATF}^{+} \mathrm{CD} 103^{+} \mathrm{DCs}$ (also called type 1 conventional DCs (cDC1s)) following exposure to chemotherapyinduced tumour debris. cDC1s are known for their ability to cross-present tumour antigens and license CD8 ${ }^{+}$ $\mathrm{T}$ cells to eliminate tumours and were required for the effect of anti-TIM3 antibodies in this model (FIG. 2). In this study, antibodies directed at HGMB1 or CEACAM1 did not facilitate CXCL9 secretion by tumour DCs, but antibodies against galectin 9 did, suggesting that blockade of galectin 9-TIM3 interactions might have antitumour potential. To gain a better understanding of the role of TIM3 in DCs, how it affects antigen cross-presentation and how it inhibits the inflammatory phenotype in autoimmunity and cancer, will require genetic experiments involving the DC-specific deletion of TIM3 using the CD11 $c^{\text {cre }}\left(\right.$ REF. $\left.^{120}\right)$, ZBTB $46^{\text {cre }}\left(\right.$ REF. $\left.^{121}\right)$ or cDC1-specific XCR $1^{\text {cre }}\left(\right.$ REF. $\left.^{122}\right)$ constructs.

In models of brain ischaemia, TIM3 is upregulated in glial cells in a hypoxia-inducible factor $1 \alpha$ (HIF1 $\alpha)$ dependent manner, and TIM3 blockade has been shown to limit infarction size and the local recruitment of neutrophils into the ischaemic lesion ${ }^{123}$. Microglia express high levels of TIM3 (REF. ${ }^{5}$ ), and both neurons ${ }^{124}$ and astrocytes ${ }^{125}$ express galectin 9 and can facilitate TIM3galectin 9 interactions with microglia. The importance of the TIM3-galectin 9 axis in the central nervous system and its importance in neurological diseases is yet to be determined. Collectively, these data support the hypothesis that TIM3 is an inhibitory receptor on myeloid cells and that modulating TIM3 signals may be of therapeutic value in treating many clinical disorders ranging from autoimmunity to cancer.

TIM3 is also expressed by mature resting CD56 dim NK cells and is further upregulated on activation in response to cytokine stimulation ${ }^{6,126}$. High expression of TIM3 is a marker of effector NK cells that are producing IFN $\gamma$ and are undergoing degranulation ${ }^{6}$. However, similarly to its expression by T cells in cancer, TIM3 might be a marker for dysfunctional NK cells. Accordingly, TIM3 blockade has been shown to reverse NK cell dysfunction in NK cells purified from the blood of patients with melanoma ${ }^{127}$.

Recent data also describe an important role for TIM3 expression in NK cells at the maternal-fetal immune interface (reviewed in REF. ${ }^{128}$ ). NK cells constitute the vast majority of the total pool of decidual immune cells $(60-80 \%)^{129}$. The numbers of $\mathrm{TIM}^{+} \mathrm{NK}$ cells are significantly increased in human placentae during the first trimester of pregnancy, and these cells exhibit a more immunosuppressive phenotype compared with TIM3 NK cells. In patients with recurrent miscarriages, these $\mathrm{TIM}^{+}$NK cells were found to have weaker immunosuppressive properties, and pregnant mice treated with anti-TIM3 antibodies have smaller litter sizes due to the disruption of maternal-fetal tolerance ${ }^{130}$. In addition to the reduced immunosuppressive capacity of $\mathrm{TIM}^{+} \mathrm{NK}$ cells in patients with recurrent miscarriages, the actual numbers of $\mathrm{TIM}^{+} \mathrm{NK}$ cells were found to decrease following miscarriage ${ }^{131}$. It has been shown that TIM3 inhibits NK cell cytotoxicity towards human embryonic trophoblasts in a manner that is dependent on galectin 9 (REF. ${ }^{132}$ ), which is expressed by decidual $\mathrm{T}_{\text {reg }}$ cells ${ }^{133}$ and/or the trophoblast cells ${ }^{131}$.

On mast cells, TIM3 has been suggested to have an activating function, which is in contrast to its role in other immune cells. Antibodies against TIM3 enhance the secretion of IL-3, IL-4, IL-6 and IL-13 in mast cells following IgE sensitization and antigen-dependent activation in vitro ${ }^{134}$. Moreover, in vitro experiments showed that both genetic deletion or siRNA knockdown of TIM3 significantly diminished IL-6 production following mast cell activation, and genetic deletion of TIM3 abrogated antigen-mediated glycolysis, an effect mediated by the FceRII receptor ${ }^{7,135}$. Further investigation is needed to decipher the role TIM3 signalling in mast cells and its importance with regard to allergies and mast cell biology.

\section{Genetic TIM3 alterations in human disease}

Polymorphisms. In humans, polymorphisms in the coding and non-coding regions of HAVCR2 have been linked to allergic diseases and autoimmunity (reviewed in REF. ${ }^{136}$ ). Three main polymorphisms in HAVCR2 have been associated with multiple clinical conditions: $-1516 \mathrm{G} / \mathrm{T}$ (rs10053538) and -574G/T (rs10515746) in the promoter region and $+4259 \mathrm{~T} / \mathrm{G}$ (rs1036199) in the coding region. These polymorphisms were associated with rhinitis ${ }^{137}$, cancer of the gastrointestinal tract ${ }^{138}$, renal cell carcinoma and pancreatic cancer ${ }^{139,140}$, nonsmall-cell lung cancer ${ }^{141}$ and in patients infected with HIV with non-Hodgkin lymphoma ${ }^{141}$. Although it is not known how these polymorphisms affect TIM3 expression and function, one study reported that two polymorphisms in PD1 (+8669AA (rs10204525)) and HAVCR2 $(-1516 \mathrm{G} / \mathrm{T})$ are correlated with high expression of TIM3 by liver-infiltrating lymphocytes in patients with hepatocellular carcinoma who are infected with $\mathrm{HBV}^{142}$. A more direct link between HAVCR2 polymorphism and $\mathrm{T}$ cell function was seen in patients with osteoarthritis carrying the HAVCR2 $+4259 \mathrm{~T} / \mathrm{G}$ allele, who showed high levels of IFN $\gamma$ production by their $\mathrm{CD}^{+} \mathrm{T}^{\mathrm{T}}$ cells ${ }^{143}$. Importantly, polymorphisms in seven amino acids in the TIM3 immunoglobulin V domain exist between the $\mathrm{T}_{\mathrm{H}} 1$ cell-biased DBA mice and $\mathrm{T}_{\mathrm{H}} 2$ cell-biased BALB/c mice, which may contribute to the differential cytokine profiles of the two mouse strains ${ }^{2}$. 
a

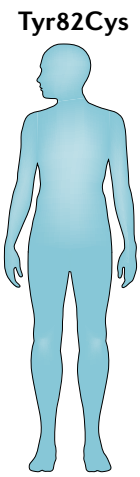

Polynesian/ East Asian

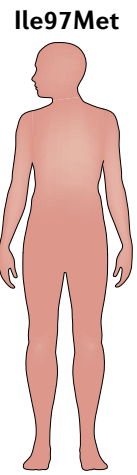

Caucasian

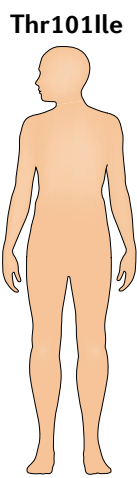

East Asian b

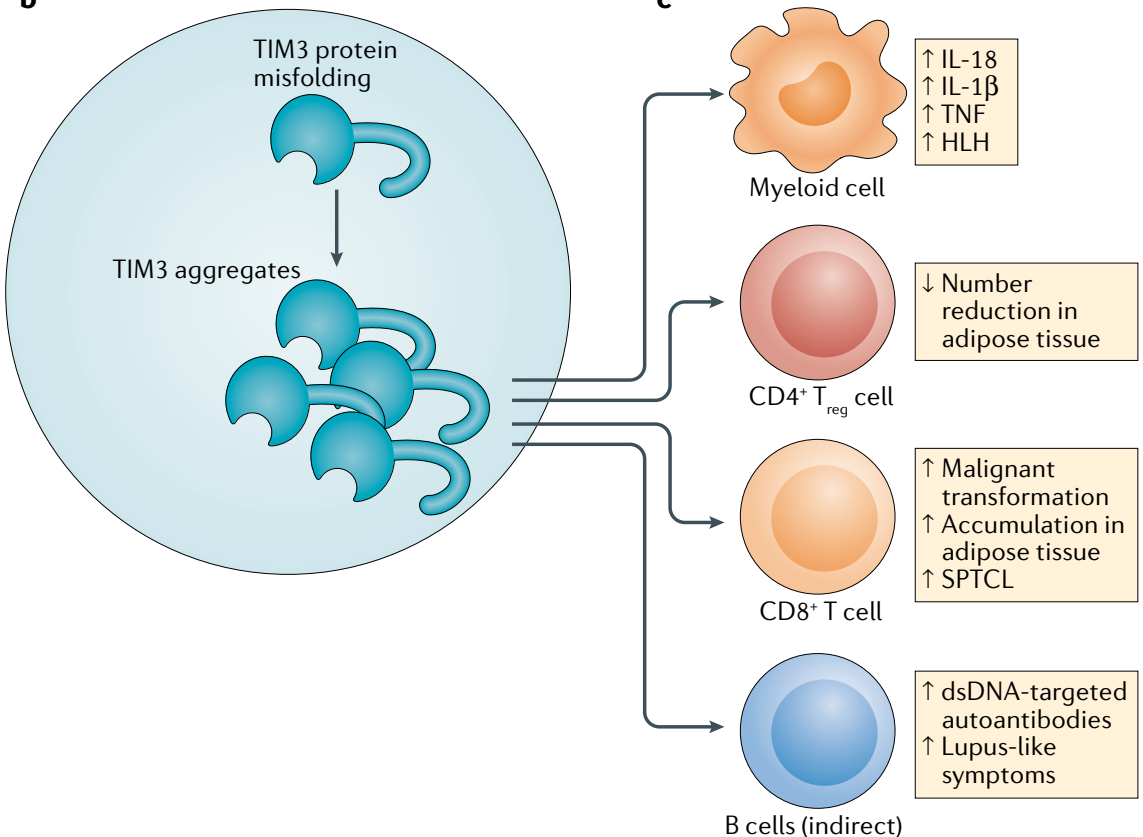

Fig. 3 | TIM3 gene single-point mutations give rise to loss of function in SPTCL. a | Three mutations in the immunoglobulin $\checkmark$ domain of T cell immunoglobulin and mucin domain-containing protein 3 (TIM3) have a different epidemiology in the general world population. The Tyr82Cys and lle97Met mutations can cause disease either as homozygous or compound mutations, and the Thr101lle mutation may cause disease as a compound mutation with the Tyr82Cys mutation, which suggests a similar function of these residues. $\mathbf{b}$ |The TIM3 mutations Tyr82Cys, lle97Met and Thr101/le lead to protein misfolding, resulting in TIM3 intracellular aggregates, which abrogate TIM3 signalling. c | Due to the broad expression and function of TIM3 in different immune cells, the absence of TIM3 signalling leads to systemic immune hyperactivation, resulting in myeloid cell dysregulation, a reduction in the number of regulatory $T$ cells $\left(T_{\text {reg }}\right.$ cells) in subcutaneous adipose tissue, excessive proliferation and malignancy of $\mathrm{CD} 8{ }^{+} \mathrm{T}$ cells in the adipose tissue, which gives rise to subcutaneous fat lesions, and an indirect promotion of autoantibody production by B cells, resulting in autoimmune symptoms with lupus-like disease. dsDNA, double-stranded DNA; HLH, haemophagocytic lymphohistiocytosis; SPTCL, subcutaneous panniculitis-like T cell lymphoma; TNF, tumour necrosis factor.

TIM3 germline mutations. With regard to the debate whether TIM3 acts as an inhibitory or a stimulatory molecule, new evidence linking germline HAVCR2 mutations to human disease indicates that TIM3 acts as an inhibitory molecule ${ }^{144-146}$. It was shown that mutations in the TIM3 immunoglobulin V domain, c. $245 \mathrm{~A}>\mathrm{G}$ (Tyr82Cys) and c.291A $>\mathrm{G}$ (Ile97Met), result in misfolding of TIM3 and loss of its expression on the cell surface of both T cells and myeloid cells. As a result of the mutation, TIM3 aggregates intracellularly. Patients harbouring these loss-of-function mutations exhibit a severe autoinflammatory and autoimmune phenotype with an excessive production of the proinflammatory molecules CXCL10, IL-1 $\beta$, IL-18 and soluble CD25. Macrophages are hyperactivated, resulting in excessive IL-1 and TNF levels in the serum and, finally, similarly to what is found in patients with systemic lupus erythematosus, B cells generate autoantibodies against double-stranded DNA. The dysregulated inflammatory state in these patients further promotes the development of subcutaneous panniculitislike T cell lymphoma (SPTCL) ${ }^{145}$ (FIG. 3), which can be associated with haemophagocytic lymphohistiocyto$\operatorname{sis}^{147,148}$. Strikingly, the onset of SPTCL is much earlier in patients with HAVCR2 mutations as compared with patients with normal HAVCR2, and the disease is more severe, with most patients with mutant TIM3 developing haemophagocytic lymphohistiocytosis.

The Tyr82Cys mutation is predominantly found in populations with Polynesian and East Asian origin, whereas the Ile97Met mutation is characteristic of patients of European origin. Sixty percent of patients with SPTCL examined possessed at least one of these mutations ${ }^{145}$. An additional study in an independent cohort of Japanese patients with SPTCL detected a homozygous Tyr82Cys mutation in 10 of 13 patients. Since the frequency of a homozygous Tyr82Cys mutation in the general population is estimated to be 1 in 35,000 , this mutation likely confers high susceptibility to SPTCL $^{146}$. Moreover, one patient with SPTCL carried a heterozygous Tyr82Cys mutation and an additional heterozygous mutation, Thr101Ile. Both studies found that HAVCR2 mutations occur mainly in patients of Asian ethnicity, which correlates with the high prevalence of known HAVCR2 single-nucleotide polymorphisms in Korean ${ }^{137}$ and Chinese $\operatorname{Han}^{138}$ populations. Importantly, the Thr101Ile mutation is a known variant of HAVCR2; when this variant is expressed ectopically in human embryonic kidney 293 (HEK293) cells, TIM3 is hypoglycosylated and remains mainly in the endoplasmic reticulum and Golgi apparatus, with minimal surface expression $^{13}$. Notably, homozygous Thr101Ile mutations 
were also found in patients with inflammatory bowel disease $^{13}$. Together these data demonstrate that a germline loss-of-function mutation in HAVCR2 results in hyperimmune activation and autoinflammatory-like disease. Although SPTCL is considered to be a T cell lymphoma, treatment with the immunosuppressive IL-1 $\beta$ inhibitor anakinra ameliorates disease symptoms, indicating that it is the excessive inflammation resulting from loss of TIM 3 that gives rise to malignancy ${ }^{145}$. These new data provide a strong indication that TIM3 is indeed an inhibitory molecule and that its aberrant folding and lack of surface expression can give rise to autoimmunity and inflammation. Whether the malignancy observed in patients is due to the excessive inflammation or vice versa is unknown at present.

How these mutations result in TIM3 misfolding is also not known. Since the mutations occur in the vicinity of predicted glycosylation sites, the Tyr82Cys and Thr101Ile mutations may both hinder glycosylation of the $\mathrm{N}$-linked or $\mathrm{O}$-linked glycosylation sites. These mutations are predicted to be in a pocket where galectin 9 is thought to bind. Since glycosylation is important for appropriate protein folding ${ }^{149}$ and stability ${ }^{150}$, one can postulate that interference with intracellular galectin 9 binding in a yet undetermined mechanism might result in the misfolding of TIM3 and thereby prevent its cellsurface expression. Another possibility is that these mutations affect the TIM3-CEACAM1 interaction, which is important for TIM3 glycosylation, maturation and trafficking to the cell surface ${ }^{13}$. The onset of SPTCL occurs usually during the fourth decade of life and might result from the accumulation of multiple inflammatory insults throughout life, which, beyond a certain threshold, result in disease. Genetically engineered animals carrying these point mutations, together with modelling and determination of the crystal structure of the aberrant, misfolded protein, will allow a better understanding of how these mutations alter TIM3 biology and its relation to SPTCL.

\section{TIM3 inhibition in the clinic}

The vast body of evidence supporting an inhibitory function for TIM3 and the prospect of TIM3 blockade enhancing anti-PD1 therapy in cancer has led to the development of anti-TIM3 blocking antibodies for clinical application. Several clinical trials using antiTIM3 antibodies in cancer patients are currently under way (TABLE 2). Most trials include a combination arm that includes anti-PD1 treatment once dose escalation and safety criteria have been met for single-agent treatment with anti-TIM3 blocking antibodies. At least one bispecific antibody targeted at both TIM3 and PD1 is being tested ${ }^{151}$. The first trial data to be reported were for TSR-022, a humanized anti-TIM3 IgG4 antibody developed by Tesaro ${ }^{152}$. Single-agent administration of TSR-022 resulted in stable disease in 31 patients and one partial response in a patient with leiomyosarcoma, with no dose-limiting toxicity observed ${ }^{153}$. This opened the door to the combination treatment with the antiPD1 antibody TSR-042. Dose escalation of TSR-022 with a fixed dose of TSR-042 showed increased clinical activity (stable disease and partial responses) correlating with increased doses of TSR-022 in 202 patients with non-small-cell lung cancer that was refractory to treatment with anti-PD1 or anti-PDL1 antibodies $^{154}$. Importantly, the combination was well tolerated, with no dose-limiting toxicity observed. Eli Lilly and Company recently reported that their TIM3 blocking antibody, LY3321367, successfully passed phase I clinical trials ${ }^{155}$, and Novartis also reported the successful completion of a phase I clinical trial with the anti-TIM3 antibody MGB453 (REF. ${ }^{156}$ ). These data are in line with

Table 2 | Ongoing and planned clinical trials with anti-TIM3 reagents

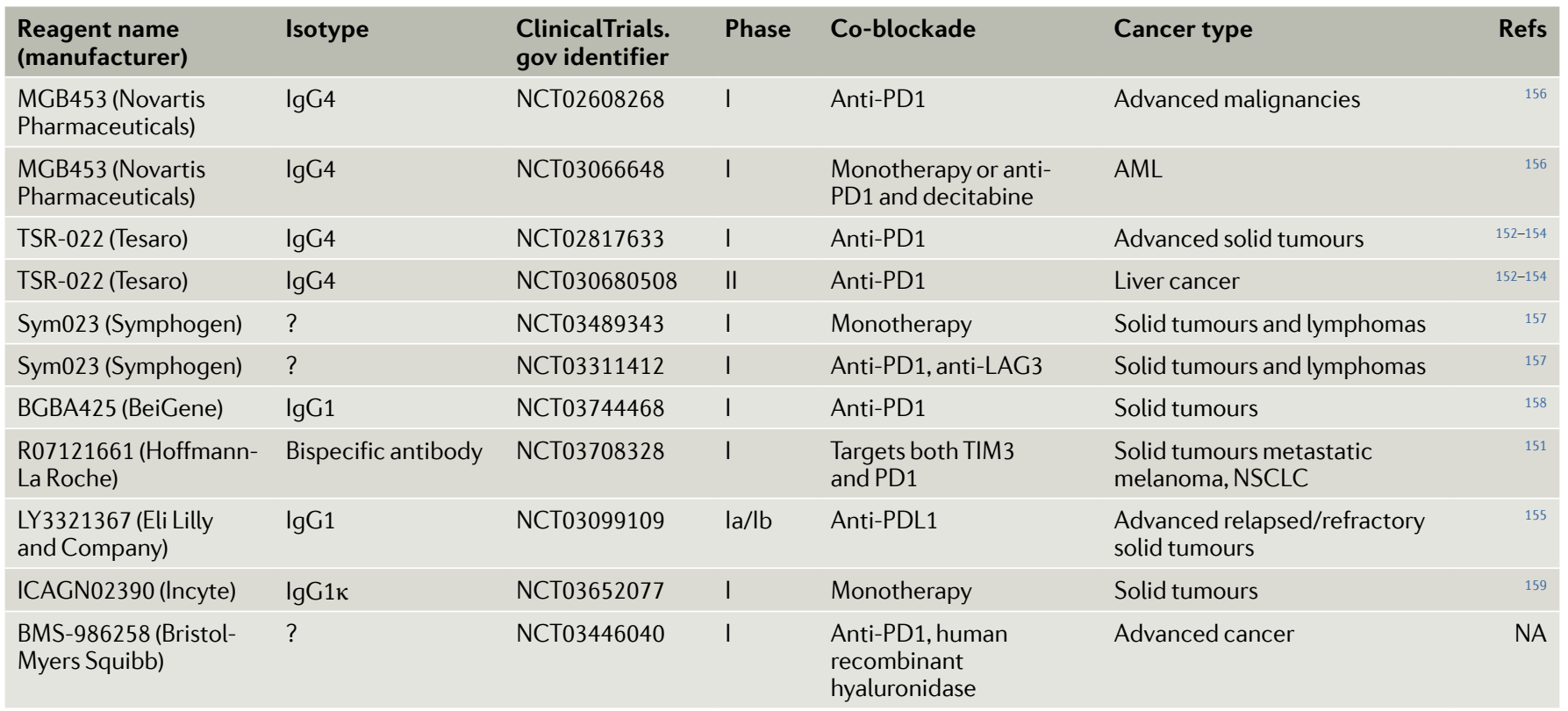

AML, acute myeloid leukaemia; LAG3, lymphocyte activation gene 3 protein; NA, not applicable; NSCLC, non-small-cell lung cancer; PD1, programmed cell death 1; PDL1, programmed cell death 1 ligand 1. 
data in preclinical models showing the superior efficacy of TIM3 pathway and PD1 pathway co-blockade over single-agent treatments ${ }^{84,97}$. Equally important, and similarly to antibodies with antitumour efficacy in preclinical models that were targeted at mouse TIM3, many of the current antibodies tested in humans are directed at the PtdSer binding site (TABLE 2).

\section{Conclusion and perspective}

TIM3 biology is complex in terms of its non-canonical signalling, broad expression by different types of immune cells and multiple ligands. Despite some debate about whether it acts as an inhibitory receptor or a stimulatory receptor, naturally occurring germline loss-of-function mutations in HAVCR2 argue for the function of TIM3 as an inhibitory receptor with a key role in regulating inflammation. Ample preclinical data support the therapeutic modulation of TIM3 in multiple disease contexts, including autoimmunity, infection, and cancer. Currently, the therapeutic potential of targeting TIM3 is being tested in cancer, where its co-blockade with other checkpoint receptors is being investigated in the clinic, with promising results in patients with anti-PD1-refractory disease. Although much remains to be learned about the circuitry by which TIM3 operates to mediate its effects in different contexts and cell types, its potential as a drug target in diverse human diseases will fuel further investigations into its biology.

Published online 1 November 2019
1. Monney, L. et al. Th1-specific cell surface protein Tim-3 regulates macrophage activation and severity of an autoimmune disease. Nature 415, 536-541 (2002) This is the first report describing cloning of TIM3 and its definition as an immunoregulatory molecule.

2. Mclntire, J. J. et al. Identification of Tapr (an airway hyperreactivity regulatory locus) and the linked Tim gene family. Nat. Immunol. 2, 1109 (2001).

3. Meyers, J. H., Sabatos, C. A., Chakravarti, S. \& Kuchroo, V. K. The TIM gene family regulates autoimmune and allergic diseases. Trends $\mathrm{Mol}$. Med. 11, 362-369 (2005).

4. Gao, X. et al. TIM-3 expression characterizes regulatory $\mathrm{T}$ cells in tumor tissues and is associated with lung cancer progression. PLOS ONE 7, e30676 (2012).

5. Anderson, A. C. et al. Promotion of tissue inflammation by the immune receptor Tim-3 expressed on innate immune cells. Science 318, 1141-1143 (2007).

6. Ndhlovu, L. C. et al. Tim-3 marks human natural killer cell maturation and suppresses cell-mediated cytotoxicity. Blood 119, 3734-3743 (2012).

7. Phong, B. L. et al. Tim-3 enhances FcepsilonRI-proximal signaling to modulate mast cell activation. J. Exp. Med. 212, 2289-2304 (2015).

8. Anderson, A. C., Joller, N. \& Kuchroo, V. K. Lag-3, Tim-3, and TIGIT: Co-inhibitory receptors with specialized functions in immune regulation. Immunity 44, 989-1004 (2016).

9. Rangachari, M. et al. Bat3 promotes T cell responses and autoimmunity by repressing Tim-3-mediated cell death and exhaustion. Nat. Med. 18, 1394-1400 (2012).

This article is the first to describe the molecular interactions of TIM3 and BAT3.

10. Lee, J. et al. Phosphotyrosine-dependent coupling of Tim-3 to T-cell receptor signaling pathways. Mol. Cell. Biol. 31, 3963-3974 (2011)

11. Clayton, K. L. et al. T cell Ig and mucin domaincontaining protein 3 is recruited to the immune synapse, disrupts stable synapse formation, and associates with receptor phosphatases. J. Immunol. 192, 782-791 (2014).

12. van de Weyer, P. S. et al. A highly conserved tyrosine of Tim-3 is phosphorylated upon stimulation by its ligand galectin-9. Biochemical biophysical Res. Commun. 351, 571-576 (2006).

13. Huang, Y. H. et al. CEACAM 1 regulates TIM-3mediated tolerance and exhaustion. Nature $\mathbf{5 1 7}$ 386-390 (2015)

14. Davidson, D., Schraven, B. \& Veillette, A PAG-associated FynT regulates calcium signaling and promotes anergy in T lymphocytes. Mol. Cell. Biol. 27, 1960-1973 (2007)

15. Avery, L., Filderman, J., Szymczak-Workman, A. L. \& Kane, L. P. Tim-3 co-stimulation promotes short-lived effector T cells, restricts memory precursors, and is dispensable for T cell exhaustion. Proc. Natl Acad. Sci. USA 115, 2455-2460 (2018).

16. Sabatos, C. A. et al. Interaction of Tim-3 and Tim-3 ligand regulates $T$ helper type 1 responses and induction of peripheral tolerance. Nat. Immunol. $\mathbf{4}$ 1102-1110 (2003).

17. Zhu, C. et al. The Tim-3 ligand galectin-9 negatively regulates T helper type 1 immunity. Nat. Immunol. 6 , 1245 (2005)

18. Clayton, K. L. et al. Soluble T cell immunoglobulin mucin domain 3 is shed from CD8+ T cells by the sheddase ADAM 10 , is increased in plasma during untreated HIV infection, and correlates with HIV disease progression. J. Virol. 89, 3723-3736 (2015).

19. Moller-Hackbarth, K. et al. A disintegrin and metalloprotease (ADAM) 10 and ADAM 17 are major sheddases of T cell immunoglobulin and mucin domain 3 (Tim-3). J. Biol. Chem. 288, 34529-34544 (2013).

20. Hansen, J. A. et al. A novel soluble form of Tim-3 associated with severe graft-versus-host disease. Biol. Blood Marrow Transplant. 19, 1323-1330 (2013).

21. Ge, W., Li, J., Fan, W., Xu, D. \& Sun, S. Tim-3 as a diagnostic and prognostic biomarker of osteosarcoma. Tumour Biol. 39, 1010428317715643 (2017).

22. Goncalves Silva, I. et al. The Tim-3-galectin-9 secretory pathway is involved in the immune escape of human acute myeloid leukemia cells. EBioMedicine $\mathbf{2 2}$ 44-57 (2017)

23. Cao, E. et al. T cell immunoglobulin mucin-3 crystal structure reveals a galectin-9-independent ligandbinding surface. Immunity 26, 311-321 (2007). This article reports the structure of TIM3 and predicted binding sites for ligands other than galectin 9 .

24. Saresella, M. et al. A role for the TIM-3/GAL-9/BAT3 pathway in determining the clinical phenotype of multiple sclerosis. FASEB J.28, 5000-5009 (2014).

25. Ju, Y. et al. The Tim-3/galectin-9 pathway involves in the homeostasis of hepatic Tregs in a mouse model of concanavalin A-induced hepatitis. Mol. Immunol. 58 , 85-91 (2014).

26. Dardalhon, V. et al. Tim-3/galectin-9 pathway: regulation of Th 1 immunity through promotion of CD $11 \mathrm{~b}+$ Ly- $6 \mathrm{G}^{+}$myeloid cells. J. Immunol. 185 1383-1392 (2010).

27. Kang, C. W. et al. Apoptosis of tumor infiltrating effector TIM-3+CD8+ T cells in colon cancer. Sci. Rep. 5, 15659 (2015)

28. Kikushige, Y. et al. A TIM-3/Gal-9 autocrine stimulatory loop drives self-renewal of human myeloid leukemia stem cells and leukemic progression. Cell Stem Cell 17, 341-352 (2015)

29. Jayaraman, P. et al. Tim3 binding to galectin-9 stimulates antimicrobial immunity. J. Exp. Med. 207, 2343-2354 (2010).

30. Elahi, S., Niki, T., Hirashima, M. \& Horton, H. Galectin-9 binding to Tim-3 renders activated human CD4+ T cells less susceptible to HIV- 1 infection. Blood 119, 4192-4204 (2012)

31. Su, E. W., Bi, S. \& Kane, L. P. Galectin-9 regulates $T$ helper cell function independently of Tim-3. Glycobiology 21, 1258-1265 (2011)

32. Oomizu, S. et al. Galectin-9 suppresses Th 17 cell development in an IL-2-dependent but Tim-3 independent manner. Clin. Immunol. 143, 51-58 (2012).

33. Wu, C. et al. Galectin-9-CD44 interaction enhances stability and function of adaptive regulatory $\mathrm{T}$ cells. Immunity 41, 270-282 (2014)

34. Leitner, J. et al. TIM-3 does not act as a receptor for galectin-9. PLOS Pathog. 9, e 1003253 (2013).

35. Freeman, G. J., Casasnovas, J. M., Umetsu, D. T. \& DeKruyff, R. H. TIM genes: a family of cell surface phosphatidylserine receptors that regulate innate and adaptive immunity. Immunol. Rev. 235, 172-189 (2010).
36. Kobayashi, N. et al. TIM-1 and TIM-4 glycoproteins bind phosphatidylserine and mediate uptake of apoptotic cells. Immunity 27, 927-940 (2007).

37. DeKruyff, R. H. et al. T cell/transmembrane, Ig, and mucin-3 allelic variants differentially recognize phosphatidylserine and mediate phagocytosis of apoptotic cells. J. Immunol. 184, 1918-1930 (2010)

38. Gandhi, A. K. et al. High resolution X-ray and NMR structural study of human T-cell immunoglobulin and mucin domain containing protein-3. Sci. Rep. 8, 17512 (2018).

39. Nakayama, M. et al. Tim-3 mediates phagocytosis of apoptotic cells and cross-presentation. Blood 113, 3821-3830 (2009).

40. Sabatos-Peyton, C. A. et al. Blockade of Tim-3 binding to phosphatidylserine and CEACAM 1 is a shared feature of anti-Tim-3 antibodies that have functional efficacy. Oncoimmunology 7, e1385690 (2018).

41. Chiba, S. et al. Tumor-infiltrating DCs suppress nucleic acid-mediated innate immune responses through interactions between the receptor TIM-3 and the alarmin HMGB1. Nat. Immunol. 13, 832-842 (2012).

42. Curtin, J. F. et al. HMGB1 mediates endogenous TLR2 activation and brain tumor regression. PLOS Med. 6 , e 10 (2009)

43. Tesniere, A. et al. Immunogenic death of colon cancer cells treated with oxaliplatin. Oncogene 29, 482-491 (2010).

44. Gray-Owen, S. D. \& Blumberg, R. S. CEACAM1: contact-dependent control of immunity. Nat. Rev. Immunol. 6, 433-446 (2006).

45. Khairnar, V. et al. CEACAM 1 promotes $C D 8^{+} \mathrm{T}$ cell responses and improves control of a chronic viral infection. Nat. Commun. 9, 2561 (2018).

46. Kammerer, R., Stober, D., Singer, B. B., Obrink, B. \& Reimann, J. Carcinoembryonic antigen-related cell adhesion molecule 1 on murine dendritic cells is a potent regulator of T cell stimulation. J. Immunol. 166, 6537-6544 (2001).

47. Horst, A. K. et al. CEACAM1 + myeloid cells control angiogenesis in inflammation. Blood 113 , 6726-6736 (2009).

48. Coutelier, J. P. et al. B lymphocyte and macrophage expression of carcinoembryonic antigen-related adhesion molecules that serve as receptors for murine coronavirus. Eur. J. Immunol. 24, 1383-1390 (1994).

49. Wiener $Z$ et al. TIM- 3 is expressed in melanoma cells and is upregulated in TGF-beta stimulated mast cells. J. Invest. Dermatol. 127, 906-914 (2007).

50. Leung, N. et al. Deletion of the carcinoembryonic antigen-related cell adhesion molecule 1 (Ceacam 1) gene contributes to colon tumor progression in a murine model of carcinogenesis. Oncogene 25, 5527-5536 (2006).

51. Song, J. H. et al. Genetic alterations and expression pattern of CEACAM1 in colorectal adenomas and cancers. Pathol. Oncol. Res 17, 67-74 (2011).

52. Golden-Mason, L. \& Rosen, H. R. Galectin-9: diverse roles in hepatic immune homeostasis and inflammation. Hepatology 66, 271-279 (2017).

53. Yan, J. et al. Tim-3 expression defines regulatory T cells in human tumors. PLOS ONE 8, e58006 (2013).

54. Khademi, M. et al. T Cell Ig- and mucin-domaincontaining molecule-3 (TIM-3) and TIM-1 molecules are differentially expressed on human Th1 and Th2 cells 
and in cerebrospinal fluid-derived mononuclear cells in multiple sclerosis. J. Immunol. 172, 7169-7176 (2004).

55. Anderson, A. C. et al. T-bet, a Th 1 transcription factor regulates the expression of Tim-3. Eur. J. immunology 40, 859-866 (2010).

56. Zhu, C. et al. An IL-27/NFIL3 signalling axis drives Tim-3 and IL-10 expression and T-cell dysfunction. Nat. Commun. 6, 6072 (2015)

57. Chihara, N. et al. Induction and transcriptional regulation of the co-inhibitory gene module in T cells Nature 558, 454-459 (2018)

This work described the IL-27 dependan co-inhibitory module in tumour infiltrating $T$ cells which include TIM3 alongside other co-inhibitory molecules.

58. Lee, S. Y. \& Goverman, J. M. The influence of T cell Ig mucin-3 signaling on central nervous system autoimmune disease is determined by the effector function of the pathogenic T cells. J. Immunol. 190 , 4991-4999 (2013)

59. Hastings, W. D. et al. TIM-3 is expressed on activated human CD4+ T cells and regulates Th 1 and Th1 7 cytokines. Eur. J. Immunol. 39, 2492-2501 (2009).

60. Shi, F. et al. Dysregulated Tim-3 expression and its correlation with imbalanced CD4 helper T cell function in ulcerative colitis. Clin. Immunol. 145, 230-240 (2012)

61. Koguchi, K. et al. Dysregulated T cell expression of TIM3 in multiple sclerosis. J. Exp. Med. 203 1413-1418 (2006)

62. Yang, L., Anderson, D. E., Kuchroo, J. \& Hafler, D. A Lack of TIM-3 immunoregulation in multiple sclerosis. J. Immunol. 180, 4409-4414 (2008).

63. Liu, Y. et al. Increased Tim-3 expression on peripheral lymphocytes from patients with rheumatoid arthritis negatively correlates with disease activity. Clin. Immunol. 137, 288-295 (2010)

64. Kanai, Y., Satoh, T., Igawa, K. \& Yokozeki, H. Impaired expression of Tim-3 on Th17 and Th1 cells in psoriasis. Acta Derm. Venereol. 92, 367-371 (2012).

65. Li, X. et al. Involvement of T cell Ig mucin-3 (Tim-3) in the negative regulation of inflammatory bowel disease Clin. Immunol. 134, 169-177 (2010)

66. Sanchez-Fueyo, A. et al. Tim-3 inhibits T helper type 1-mediated auto- and alloimmune responses and promotes immunological tolerance. Nat. Immunol. 4 1093-1101 (2003)

67. Wang, F. et al. The Tim-3 ligand galectin-9 negatively regulates $\mathrm{CD}^{+}$alloreactive $\mathrm{T}$ cell and prolongs survival of skin graft. Cell. Immunol. 250, 68-74 (2007)

68. Sakuishi, $\mathrm{K}$ et al. TIM3 ${ }^{+} \mathrm{FOXP3}^{+}$regulatory $\mathrm{T}$ cells are tissue-specific promoters of T-cell dysfunction in cancer. Oncoimmunology 2, e23849 (2013).

69. Gupta, S. et al. Allograft rejection is restrained by short-lived TIM-3+PD-1+Foxp3+ $\mathrm{T}_{\text {regs }}$ J. Clin. Invest. 122, 2395-2404 (2012).

70. Henao-Tamayo, M., Irwin, S. M., Shang, S., Ordway, D. $\&$ Orme, I. M. T lymphocyte surface expression of exhaustion markers as biomarkers of the efficacy of chemotherapy for tuberculosis. Tuberculosis 91 308-313 (2011)

71. Cyktor, J. C., Carruthers, B., Beamer, G. L. \& Turner, J. Clonal expansions of CD8+ T cells with IL-10 secreting capacity occur during chronic Mycobacterium tuberculosis infection. PLOS ONE 8, e58612 (2013)

72. Wang, $X$ et al. Elevated expression of Tim-3 on CD8 $T$ cells correlates with disease severity of pulmonary tuberculosis. J. Infect. 62, 292-300 (2011).

73. Qiu, Y. et al. Tim-3-expressing CD4+ and CD8+ T cells in human tuberculosis (TB) exhibit polarized effector memory phenotypes and stronger anti-TB effector functions. PLOS Pathog. 8, e1002984 (2012).

74. Jayaraman, P. et al. IL-1 beta promotes antimicrobial immunity in macrophages by regulating TNFR signaling and caspase-3 activation. J. Immunol. 190 4196-4204 (2013).

75. Sada-Ovalle, I. et al. The Tim3-galectin 9 pathway induces antibacterial activity in human macrophages infected with Mycobacterium tuberculosis. J. Immunol. 189, 5896-5902 (2012).

76. Hashimoto, M. et al. CD8 T cell exhaustion in chronic infection and cancer: opportunities for interventions. Annu. Rev. Med 69, 301-318 (2018).

77. Jones, R. B. et al. Tim-3 expression defines a nove population of dysfunctional T cells with highly elevated frequencies in progressive HIV-1 infection. J. Exp. Med. 205, 2763-2779 (2008)

78. McMahan, R. H. et al. Tim-3 expression on PD- $1^{+}$HCV specific human CTLs is associated with viral persistence, and its blockade restores hepatocyte-directed in vitro cytotoxicity. J. Clin. Invest. 120, 4546-4557 (2010).
79. Jin, H. T. et al. Cooperation of Tim-3 and PD-1 in CD8 T-cell exhaustion during chronic viral infection. Proc. Natl Acad. Sci. USA 107, 14733-14738 (2010).

80. Ju, Y. et al. Blockade of Tim-3 pathway ameliorates interferon-gamma production from hepatic CD8+ $T$ cells in a mouse model of hepatitis B virus infection. Cell. Mol. Immunol. 6, 35-43 (2009).

81. Takamura, S. et al. Premature terminal exhaustion of Friend virus-specific effector $\mathrm{CD} 8^{+} \mathrm{T}$ cells by rapid induction of multiple inhibitory receptors. J. Immunol. 184, 4696-4707 (2010)

82. Wu, W. et al. Tim-3 expression on peripheral T cell subsets correlates with disease progression in hepatitis B infection. Virol. J. 8, 113 (2011).

83. Fourcade, J. et al. PD-1 and Tim-3 regulate the expansion of tumor antigen-specific CD8(+) T cells induced by melanoma vaccines. Cancer Res. $\mathbf{7 4}$, 1045-1055 (2014)

84. Sakuishi, K. et al. Targeting Tim-3 and PD-1 pathways to reverse $\mathrm{T}$ cell exhaustion and restore anti-tumor immunity. J. Exp. Med. 207, 2187-2194 (2010). References 83 and 84 demonstrate the efficacy of combined anti-PD1 and anti-TIM3 immunotherapy for solid tumours.

85. Fourcade, J. et al. Upregulation of Tim-3 and PD-1 expression is associated with tumor antigen-specific $\mathrm{CD} 8+\mathrm{T}$ cell dysfunction in melanoma patients. J. Exp. Med. 207, 2175-2186 (2010).

86. Liu, J. F. et al. Blockade of TIM3 relieves immunosuppression through reducing regulatory T cells in head and neck cancer. J. Exp. Clin. Cancer Res. 37, 44 (2018)

87. Zhang, Y., Cai, P., Liang, T., Wang, L. \& Hu, L. TIM-3 is a potential prognostic marker for patients with solid tumors: a systematic review and meta-analysis. Oncotarget 8, 31705-31713 (2017).

88. $\mathrm{Xu}, \mathrm{B}$. et al. Circulating and tumor-infiltrating Tim-3 in patients with colorectal cancer. Oncotarget 6 20592-20603 (2015)

89. Li, H. et al. Tim-3/galectin-9 signaling pathway mediates T-cell dysfunction and predicts poor prognosis in patients with hepatitis B virus-associated hepatocellular carcinoma. Hepatology 56, 1342-135 (2012).

90. Kikushige, Y. \& Miyamoto, T. TIM-3 as a novel therapeutic target for eradicating acute myelogenous leukemia stem cells. Int. J. Hematol. 98, 627-633 (2013).

91. Kikushige, Y. \& Miyamoto, T. Identification of TIM-3 as a leukemic stem cell surface molecule in primary acute myeloid leukemia. Oncology 89 (Suppl 1), 28-32 (2015).

92. Jan, M. et al. Prospective separation of normal and leukemic stem cells based on differential expression of TIM3, a human acute myeloid leukemia stem cell marker. Proc. Natl Acad. Sci. USA 108, 5009-5014 (2011).

93. Haubner, S. et al. Coexpression profile of leukemic stem cell markers for combinatorial targeted therapy in AML. Leukemia 33, 64-74 (2019).

94. Kikushige, $\mathrm{Y}$, et al. TIM-3 is a promising target to selectively kill acute myeloid leukemia stem cells. Cell Stem Cell 7, 708-717 (2010).

95. Koyama, S. et al. Adaptive resistance to therapeutic PD-1 blockade is associated with upregulation of alternative immune checkpoints. Nat. Commun. 7 10501 (2016)

This work highlights TIM3 upregulation as a compensatory mechanism associated with resistance to anti-PD1 therapy, which may explain the efficacy of combined anti-PD1 and anti-TIM3 immunotherapy.

96. Ngiow, S. F. et al. Anti-TIM3 antibody promotes T cell IFN-gamma-mediated antitumor immunity and suppresses established tumors. Cancer Res. 71 3540-3551 (2011).

97. Liu, J. et al. Targeting PD-1 and Tim-3 pathways to reverse CD8 T-cell exhaustion and enhance ex vivo T-cell responses to autologous dendritic/tumor vaccines. J. Immunother. 39, 171-180 (2016)

98. Zhou, Q et al. Coexpression of Tim-3 and PDidentifies a $\mathrm{CD}^{+}{ }^{+}$-cell exhaustion phenotype in mice with disseminated acute myelogenous leukemia. Blood 117, 4501-4510 (2011).

99. Golden-Mason, L. et al. Negative immune regulator Tim-3 is overexpressed on T cells in hepatitis c virus infection and its blockade rescues dysfunctional CD4 and CD8 ${ }^{+}$T cells. J. Virol. 83, 9122-9130 (2009).

100. Nebbia, G. et al. Upregulation of the Tim-3/galectin-9 pathway of T cell exhaustion in chronic hepatitis B virus infection. PLOS ONE 7, e47648 (2012).
101. Tivol, E. A. et al. CTLA-4 deficient mice exhibit massive lymphoproliferation and multi-organ lymphatic infiltration: a critical negative immunoregulatory role of CTLA-4. Immunity 3, 541-547 (1995).

102. Waterhouse, P. W. et al. Lymphoproliferative disorders with early lethality in mice deficient in CTLA-4. Science 270, 985-988 (1995).

103. Nishimura, H., Nose, M., Hiai, H., Minato, N. \& Honjo, T Development of lupus-like autoimmune diseases by disruption of the PD-1 gene encoding an ITIM motifcarrying immunoreceptor. Immunity 11, 141-151 (1999).

104. Nishimura, H. et al. Autoimmune dilated cardiomyopathy in PD-1 receptor-deficient mice. Science 291, 319-322 (2001).

105. Sade-Feldman, M. et al. Defining T cell states associated with response to checkpoint immunotherapy in melanoma. Cell 175, 998-1013 e1020 (2018).

106. Im, S. J. et al. Defining CD8 ${ }^{+} \mathrm{T}$ cells that provide the proliferative burst after PD-1 therapy. Nature 537 417-421 (2016)

This work reports the first observation of the inverse relationship between TIM3, TCF1, and $T$ cell exhaustion and stemness.

107. Kurtulus, S. et al. Checkpoint blockade immunotherapy induces dynamic changes in PD-1 CD8 ${ }^{+}$tumorinfiltrating T cells. Immunity 50, 181-194 e186 (2019).

108. Wu, T. et al. The TCF1-Bcl6 axis counteracts type I interferon to repress exhaustion and maintain $\mathrm{T}$ cell stemness. Sci. Immunol. 1, eaai8593 (2016).

109. Siddiqui, I. et al. Intratumoral Tcf $1+$ PD $-1+C D 8+T$ cells with stem-like properties promote tumor control in response to vaccination and checkpoint blockade immunotherapy. Immunity 50, 195-211.e10 (2019).

110. Brummelman, J. et al. High-dimensional single cell analysis identifies stem-like cytotoxic $\mathrm{CD} 8^{+} \mathrm{T}$ cells infiltrating human tumors. J. Exp. Med. 215 2520-2535 (2018).

111. Miller, B. C. et al. Subsets of exhausted CD8 ${ }^{+}$T cells differentially mediate tumor control and respond to checkpoint blockade. Nat. Immunol. 20, 326-336 (2019).

112. Zhang, Y. et al. Tim-3 regulates pro- and antiinflammatory cytokine expression in human CD 14 monocytes. J. Leukoc. Biol. 91, 189-196 (2012).

113. Yang, X. et al. T cell Ig mucin-3 promotes homeostasis of sepsis by negatively regulating the TLR response. J. Immunol. 190, 2068-2079 (2013).

114. Jiang, X. et al. Tim-3 promotes intestinal homeostasis in DSS colitis by inhibiting M1 polarization of macrophages. Clin. Immunol. 160, 328-335 (2015).

115. Zhao, J. et al. Human pregnancy up-regulates Tim-3 in innate immune cells for systemic immunity. J. Immunol. 182, 6618-6624 (2009).

116. Jiang, X. et al. Tim-3 promotes tumor-promoting M2 macrophage polarization by binding to STAT 1 and suppressing the STAT 1-miR-155 signaling axis. Oncoimmunology 5, e1211219 (2016).

117. Wang, Z. et al. Tim-3 inhibits macrophage control of Listeria monocytogenes by inhibiting Nrf2. Sci. Rep. 7 , 42095 (2017)

118. Miyanishi, M. et al. Identification of Tim4 as a phosphatidylserine receptor. Nature 450, 435-439 (2007).

119. de Mingo Pulido, A. et al. TIM-3 regulates CD 103+ dendritic cell function and response to chemotherapy in breast cancer. Cancer cell 33,60-74 e66 (2018). This work describes a non-T cell role for TIM3 in facilitating antitumour responses.

120. Caton, M. L., Smith-Raska, M. R. \& Reizis, B. NotchRBP-J signaling controls the homeostasis of CD8 dendritic cells in the spleen. J. Exp. Med. 204 1653-1664 (2007).

121. Loschko, J. et al. Absence of MHC class II on CDCs results in microbial-dependent intestinal inflammation. J. Exp. Med. 213, 517-534 (2016).

122. Mattiuz, R. et al. Novel Cre-expressing mouse strains permitting to selectively track and edit type 1 conventional dendritic cells facilitate disentangling their complexity in vivo. Front. Immunol. 9, 2805 (2018).

123. Koh, H. S. et al. The HIF-1/glial TIM-3 axis controls inflammation-associated brain damage under hypoxia. Nat. Commun. 6, 6340 (2015)

124. Wang, H. W., Zhu, X. L., Qin, L. M., Qian, H. J. $\&$ Wang, Y. Microglia activity modulated by $T$ cell Ig and mucin domain protein 3 (Tim-3). Cell. Immunol. 293, 49-58 (2015).

125. Steelman, A. J. \& Li, J. Astrocyte galectin-9 potentiates microglial TNF secretion. J. Neuroinflammation 11, 144 (2014) 
126. Gleason, M. K. et al. Tim-3 is an inducible human natural killer cell receptor that enhances interferon gamma production in response to galectin-9. Blood 119, 3064-3072 (2012).

127. da Silva, I. P. et al. Reversal of NK-cell exhaustion in advanced melanoma by Tim-3 blockade. Cancer Immunol. Res. 2, 410-422 (2014).

128. Miko, E., Meggyes, M., Doba, K., Barakonyi, A. $\&$ Szereday, L. Immune checkpoint molecules in reproductive immunology. Front. Immunol. 10 (2019)

129. Xu, Y. Y. Wang, S. C., Li, D. J. \& Du, M. R. Co-signaling molecules in maternal-fetal immunity. Trends Mol. Med. 23, 46-58 (2017).

130. Li, Y. et al. Tim-3 signaling in peripheral NK cells promotes maternal-fetal immune tolerance and alleviates pregnancy loss. Sci. Signal. 10, eaah4323 (2017).

131. Li, Y. H. et al. The Galectin-9/Tim-3 pathway is involved in the regulation of NK cell function at the maternalfetal interface in early pregnancy. Cell. Mol. Immunol. 13, 73-81 (2016).

132. Sun, J. et al. Tim-3 Is upregulated in NK cells during early pregnancy and inhibits NK cytotoxicity toward trophoblast in galectin-9 dependent pathway. PLOS ONE 11, e0147186 (2016).

133. Meggyes, $M$. et al. Feto-maternal immune regulation by TIM-3/galectin-9 pathway and PD-1 molecule in mice at day 14.5 of pregnancy. Placenta 36 , 1153-1160 (2015)

134. Nakae, S. et al. TIM-1 and TIM-3 enhancement of Th2 cytokine production by mast cells. Blood $\mathbf{1 1 0}$ 2565-2568 (2007).

135. Phong, B., Avery, L., Menk, A. V., Delgoffe, G. M. $\&$ Kane, L. P. Cutting edge: murine mast cells rapidly modulate metabolic pathways essential for distinct effector functions. J. Immunol. 198, 640-644 (2017).

136. Lee, J., Phong, B., Egloff, A. M. \& Kane, L. P. TIM polymorphisms-genetics and function. Genes Immun. 12, 595-604 (2011)

137. Chae, S. C., Park, Y. R., Lee, Y. C., Lee, J. H. \& Chung, H. T. The association of TIM-3 gene polymorphism with atopic disease in Korean population. Hum. Immunol. 65, 1427-1431 (2004).

138. Gao, X., Yang, J., He, Y. \& Zhang, J. Quantitative assessment of TIM-3 polymorphisms and cancer risk in Chinese Han population. Oncotarget 7 , 35768-35775 (2016)

139. Cai, C. et al. T-cell immunoglobulin- and mucin-domaincontaining molecule 3 gene polymorphisms and renal cell carcinoma. DNA Cell Biol. 31, 1285-1289 (2012).

140. Tong, D. et al. T cell immunoglobulin- and mucindomain-containing molecule 3 gene polymorphisms and susceptibility to pancreatic cancer. Mol. Biol. Rep 39, 9941-9946 (2012).

141. Bai, J. et al. T-cell immunoglobulin- and mucin-domain containing molecule 3 gene polymorphisms and prognosis of non-small-cell lung cancer. Tumour Biol. 34, 805-809 (2013).

142. Li, Z. et al. Immune checkpoint proteins PD-1 and TIM-3 are both highly expressed in liver tissues and correlate with their gene polymorphisms in patients with HBV-related hepatocellular carcinoma. Medicine 95 e5749 (2016)

143. Li, S. et al. TIM-3 genetic variations affect susceptibility to osteoarthritis by interfering with interferon gamma in $\mathrm{CD}^{+} \mathrm{T}$ cells. Inflammation 38, 1857-1863 (2015).

144. Dixon, K. O., Das, M. \& Kuchroo, V. K. Human disease mutations highlight the inhibitory function of TIM-3. Nat. Genet. 50, 1640-1641 (2018).

145. Gayden, T. et al. Germline HAVCR2 mutations altering TIM-3 characterize subcutaneous panniculitis-like T cell lymphomas with hemophagocytic lymphohistiocytic syndrome. Nat. Genet. 50, 1650-1657 (2018).

146. Polprasert, C. et al. Frequent germline mutations of HAVCR2 in sporadic subcutaneous panniculitis-like T-cell lymphoma. Blood Adv. 3, 588-595 (2019) References 145 and 146 describe single-point mutations in the HAVCR2 gene that predispose individuals to disease.

147. Gonzalez, C. L., Medeiros, L. J., Braziel, R. M. \& Jaffe, E. S. T-cell lymphoma involving subcutaneous tissue. A clinicopathologic entity commonly associated with hemophagocytic syndrome. Am. J. Surg. Pathol. 15, 17-27 (1991)

148. Pincus, L. B. et al. Subcutaneous panniculitis-like T-cell lymphoma with overlapping clinicopathologic features of lupus erythematosus: coexistence of 2 entities? Am. J. dermatopathology 31, 520-526 (2009).

149. Shental-Bechor, D. \& Levy, Y. Effect of glycosylation on protein folding: a close look at thermodynamic stabilization. Proc. Natl Acad. Sci. USA 105 8256-8261 (2008).

150. Sola, R. J. \& Griebenow, K. Effects of glycosylation on the stability of protein pharmaceuticals. J. Pharm. Sci. 98, 1223-1245 (2009)

151. Klein, C. et al. Engineering therapeutic bispecific antibodies using CrossMab technology. Methods 154 21-31 (2019).

152. Murtaza, A et al. Discovery of TSR-022, a novel, potent anti-human TIM-3 therapeutic antibody. Eur. J. Cancer 69, S102 (2016)

153. Weiss, G. J. et al. A phase 1 study of TSR-022, an anti-TIM-3 monoclonal antibody, in patients (pts) with advanced solid tumors. J. Immunother. Cancer 5, 86 (2017).
154. Davar, D. et al. A phase 1 study of TSR-022, an antiTIM-3 monoclonal antibody, in combination with TSR-042 (anti-PD-1) in patients with colorectal cance and post-PD-1 NSCLC and melanoma. J. Immunother. Cancer 6, 115 (2018)

155. Harding J. et al. A phase la/lb study of an anti-TIM-3 antibody (LY3321367) monotherapy or in combination with an anti-PD-L1 antibody (LY3300054): interim safety, efficacy, and pharmacokinetic findings in advanced cancers. J. Clin. Oncol. 37 (Suppl. 8), 12 (2019).

156. Ahn, M. Combination IO+IO. J. Thorac. Oncol. 13, S299-S300 (2018).

157. Lindsted, T. et al. Preclinical characterization of Sym023 a human anti-TIM3 antibody with a novel mechanism of action [abstract 5629]. Cancer Res. 78 5629-5629 (2018).

158. Zhang, T. et al. BGB-A425: a humanized anti-human Tim-3 antibody that exhibits strong immune cell activation [abstract 2628]. Cancer Res. 77 , 2628-2628 (2017).

159. Waight, J. et al. INCAGN02390, a novel antagonist antibody that targets the co-inhibitory receptor TIM-3 [abstract 3825]. Cancer Res. 78, 3825-3825 (2018).

\section{Acknowledgements}

This work was supported by grants from the US National Institutes of Health (R01 NS045937 to V.K.K., P01AI073748, P01AI039671 and P01AI056299 to V.K.K. and A.C.A. and R01CA187975 to A.C.A). Y.W. is supported by a Human Frontier Science Program postdoctoral fellowship (LT000019/2018).

\section{Author contributions}

The authors contributed equally to all aspects of the article.

\section{Competing interests}

A.C.A. is a member of the scientific advisory boards for Compass Therapeutics, Tizona Therapeutics and Zumutor Biologics, which have interests in cancer immunotherapy. A.C.A. is also a paid consultant for Aximmune. V.K.K. has an ownership interest in and is a member of the scientific advisory board for Tizona Therapeutics. V.K.K. is a co-founder of and has an ownership interest in Celsius Therapeutics. A.C.A.'s and V.K.K.'s interests were reviewed and managed by the Brigham and Women's Hospital and Partners Healthcare in accordance with their conflict of interest policies. A.C.A. and V.K.K. are named inventors on patents related to TIM3.

\section{Publisher's note}

Springer Nature remains neutral with regard to jurisdictional claims in published maps and institutional affiliations. 